\title{
Analyse de la flore des Alpes. 5: milieux et phytosociologie
}

\author{
David Aeschimann, Nathalie Rasolofo \& Jean-Paul Theurillat
}

\begin{abstract}
AESCHIMANN, D., N. RASOLOFO \& J.-P. THEURILLAT (2013). Analysis of the flora of the Alps. 5: habitats and phytosociology. Candollea 68: 5-27. In French, English and French abstracts.

Last in a series, this paper concludes the statistical analysis of the data published in «Flora alpina», treating that which concerns habitats and phytosociology. Expressed as percentages, the results concerning the flora are envisaged globally, by vegetation belt, by administrative division, by floristic contingent, by family, by genus and by biological form. More than $81 \%$ of endemics are found in rocky habitats, a proportion that increases with altitude, from $39 \%$ to $97 \%$ between the colline and the nival belts. Very stenoecious, $27 \%$ of the endemic taxa are exclusive of one of the major habitats, while this percentage drops to $16 \%$ among non-endemic indigenous taxa. Xenophytes are very nitrophilous: $95 \%$ of them are present in these, often anthropogenic, habitats and more than $38 \%$ are exclusive of them. Phytosociology confirms the stenoecity of endemics, as well as most of the other trends that are strongly correlated but not parallel. Cartographic patterns of four sub-alliances endemic to the Alps are provided: Physoplexido-Potentillenion, Saxifragenion lingulatae, Saxifragenion pedemontanae and Caricenion austroalpinae. The presence of many endemic taxa in these syntaxa, some narrowly stenoecious, justifies their phytosociological individualization.
\end{abstract}

\section{Key-words}

Flora of the Alps - Alpine arc - Floristics - Habitats - Phytosociology - Vegetation belts - Biological forms

\begin{abstract}
Résumé
AESCHIMANN, D., N. RASOLOFO \& J.-P. THEURILLAT (2013). Analyse de la flore des Alpes. 5: milieux et phytosociologie. Candollea 68: 5-27. En français, résumés anglais et français.

Dernier de sa série, cet article clôt l'analyse statistique des données du «Flora alpina», en traitant celles relatives aux milieux et à la phytosociologie. Exprimés en pourcentages, les résultats concernent la flore envisagée globalement, par étage de végétation, par division administrative, par contingent floristique, par famille, par genre et par forme biologique. Plus de $81 \%$ des endémiques sont présents dans les milieux rocheux, une proportion qui augmente avec l'altitude, passant de $39 \%$ à $97 \%$ de l'étage collinéen au nival. Très sténoèces, $27 \%$ des endémiques sont exclusifs d'un des principaux milieux, alors que ce pourcentage tombe à $16 \%$ chez les indigènes non endémiques. Les xénophytes sont très nitrophiles, car 95\% d'entre eux sont présents dans ces milieux souvent anthropiques et plus de $38 \%$ en sont exclusifs. La phytosociologie confirme la sténoécité des endémiques, ainsi que la plupart des autres tendances, fortement corrélées mais non parallèles. Les patrons cartographiques de quatre sous-alliances endémiques des Alpes sont fournis: Physoplexido-Potentillenion, Saxifragenion lingulatae, Saxifragenion pedemontanae et Caricenion austroalpinae. La présence de nombreux endémiques dans ces syntaxons, dont certains étroitement sténoèces, justifie leur individualisation phytosociologique.
\end{abstract}

Adresses des auteurs: DA: Conservatoire et Jardin botaniques de la Ville de Genève, Laboratoire universitaire de Systématique végétale et Biodiversité, CP 60, 1292 Chambésy, Switzerland. Email: david.aeschimann@ville-ge.ch

NR: Planta 47, 1223 Cologny, Switzerland

JPT: Fondation J.-M. Aubert, CP 71, 1938 Champex-Lac, Switzerland - Laboratoire de Biogéographie, Section de Biologie, Université de Genève, CP 60, 1292 Chambésy, Switzerland

Soumis le 3 septembre 2012. Accepté le 15 octobre 2012.

Edité par P. Bungener 


\section{Introduction}

Cet article clôt une série (AEschimann \& al., 2011a, b; 2012a, b) consacrée à l'analyse statistique des données publiées dans le «Flora alpina» (AEschimANN \& al., 2004), où les matières concernant la nomenclature, la biodiversité, la chorologie, la biologie, la phénologie et l'écologie ont déjà été abordées. Ce cinquième travail est consacré aux rubriques 18 et 19 des fiches $\mathrm{du}$ «Flora alpina» $(1: 28-33)$, qui concernent les milieux et la phytosociologie de chaque taxon. L'objectif est ici de répondre aux questions suivantes:

1. Quels sont les pourcentages de taxons potentiellement présents dans chaque milieu, en considérant la flore globalement, par étage de végétation, par contingent floristique, par famille, par genre et par forme biologique? Comment interpréter ces résultats?

2. Quels sont les pourcentages de taxons ayant leur optimum phytosociologique dans chaque syntaxon, en considérant la flore globalement et par étage de végétation? Par division administrative, quels sont les nombres de taxons endémiques ayant leur optimum dans quatre sous-alliances endémiques des Alpes? Comment interpréter ces résultats?

\section{Matériel et méthodes}

Les informations générales concernant cette série d'analyses de la Flore des Alpes ont été fournies précédemment (AESCHIMANN \& al., 2011a: 28-31), notamment celles relatives à la dition et ses divisions, aux taxons considérés et à la base de données. Les informations correspondant aux étages de végétation, ainsi qu'aux distributions mondiales des taxons, à leurs formes biologiques et à leur écologie figurent dans les $2^{\mathrm{e}}, 3^{\mathrm{e}}$ et $4^{\mathrm{e}}$ articles (AESCHIMANN \& al., 2011b: 226-228; 2012a: 6; 2012b: 194). Seuls les renseignements spécifiques au présent travail sont donnés ci-dessous.

Dans cet article, l'ensemble des 4485 taxons recensés dans le «Flora alpina» est considéré. Les résultats sont exprimés en pourcentages du total de taxons d'un groupe étudié, ce qui permet chaque fois de calculer le nombre de taxons concernés par une catégorie de milieux ou par un syntaxon.

\section{Milieux}

Les neuf grandes catégories de milieux retenues dans le «Flora alpina» $(1: 29-32)$ au niveau supérieur sont réduites à sept catégories principales pour les présentes analyses. Ce sont dès lors les milieux:

- aquatiques (code «Flora alpina» 1 : milieux aquatiques s.l. (permanents, temporaires));

- nitrophiles (2: végétation \pm nitrophile terrestre, naturelle ou anthropogène);
- rocheux (3: affleurements rocheux, sables, tuf, rochers, éboulis, gravières, carrières);

- marécageux (4 et 5: sources, ruisseaux, bas-marais s.l., tourbières);

- herbacés (6 et 7.2: prés, pâturages, pelouses, combes à neige, lisières forestières (végétation herbacée), mégaphorbiées s.1.);

- buissonneux (7.1, 7.3 à 7.6 et 8: végétation ligneuse basse: landes, garrigues, buissons s.1., brousses s.1.);

- forestiers (9: forêts).

Les 42 catégories de milieux retenues dans le «Flora alpina» au niveau intermédiaire sont réduites pour les présentes analyses, de manière à obtenir 35 catégories secondaires, listées dans les tableaux relatifs. Les milieux de niveau intermédiaire suivants sont dès lors fusionnés (codes «Flora alpina»): 1.1 à $1.3,2.2$ et $2.4,2.7$ à $2.8,3.1$ et $3.5,4.1$ à $4.2,6.7$ et 2.3 .

Les catégories de niveau inférieur retenues dans le «Flora alpina» ne sont pas prises en considération dans ce travail. D'autre part, aucune distinction n'est faite ici entre les milieux les plus importants (sans parenthèses dans le «Flora alpina») et ceux de moindre importance ou occasionnels (entre parenthèses). Les indications «?» (milieu à confirmer), [e] (épiphyte) et [p] (parasite) sont omises.

La plupart des taxons pouvant se rencontrer dans plusieurs catégories de milieux, les données générales sont complétées par les décomptes des taxons exclusifs de chaque catégorie, c'est-à-dire dont la présence est entièrement limitée à une seule catégorie. L'ensemble des milieux cités dans le «Flora alpina» pour un taxon définit sa niche réalisée sur tout l'arc alpin, la niche réalisée locale pouvant être plus petite en fonction des régions ou de l'altitude. Les pourcentages présentés ci-après sont donc relatifs à des présences potentielles et non pas effectives dans chaque situation. Ces résultats correspondent à des situations théoriques et doivent être interprétés avec discernement, en particulier dans les analyses par étage de végétation, tous les milieux qu'un taxon pouvant occuper ne se rencontrant pas nécessairement à chaque étage.

\section{Phytosociologie}

Le système phytosociologique de référence est publié dans le «Flora alpina» (3: 301-317). Il est basé sur le synsystème des Alpes de Theurillat \& al. (1995), revu et légèrement modifié, où la codification utilisée pour chaque syntaxon est également indiquée. Les 50 classes, 92 ordres, 182 alliances et 52 sous-alliances retenus sont étudiés dans ce travail. En revanche, les trois sous-classes et les 14 formations végétales établies pour le regroupement des classes ne sont pas prises en considération. 
Les résultats phytosociologiques constituent un véritable complément à ceux relatifs aux milieux, puisqu'un seul syntaxon, considéré comme l'optimum sociologique, est indiqué pour chaque taxon dans le «Flora alpina». Pour seulement douze taxons, deux syntaxons y sont indiqués comme optimum: Carex ornithopoda, C. sempervirens, Cyperus glomeratus, Dactylorhiza maculata, Epipactis atrorubens, Erigeron alpinus, Molinia arundinacea, Orobanche reticulata, Pinus sylvestris, Polygonum viviparum, Taraxacum alpinum et T. aquilonare. La plupart des taxons débordent de l'optimum sociologique attribué, même lorsque celui-ci est une classe (syntaxon entre parenthèses dans le «Flora alpina»). De ce fait, l'optimum sociologique pour un taxon de basse altitude pourra être différent à sa limite altitudinale supérieure et vice-versa. Comme pour les milieux, les pourcentages indiqués par étage de végétation sont donc théoriques et leur interprétation requiert ici aussi une certaine prudence.

\section{Résultats et discussions}

\section{Milieux}

\section{Données générales}

Parmi les 3482 taxons indigènes non endémiques recensés dans le «Flora alpina», $70 \%$ sont potentiellement présents dans les milieux herbacés et près de $57 \%$ dans les milieux nitrophiles (tableau 1: catégories principales). Les premier et second rangs occupés par ces deux catégories confirment les résultats obtenus par DAINESE \& BRAGAZZA $(2012: 13,15)$ dans la partie italienne des Alpes (AEschimann \& al., 2011a: 28). Les taux de présence sont compris entre $41 \%$ et $45 \%$ dans les milieux rocheux, buissonneux et forestiers, et entre $12 \%$ et $16 \%$ seulement dans les milieux aquatiques et marécageux. Les pourcentages de taxons exclusifs sont très faibles, le maximum étant atteint dans les milieux nitrophiles avec 5,4\%. Au total, seulement $16,1 \%$ des taxons indigènes (non endémiques) sont exclusifs d'une des sept catégories principales de milieux. Globalement, les proportions du tableau 1 sont comparables à celles relevées en Corse par JEANMONOD \& al. (2011a: 17), où le pourcentage de taxons dans les milieux herbacés est aussi le plus élevé.

Les 501 taxons endémiques sont nettement plus sténoèces que les indigènes (non endémiques), puisque $81,2 \%$ d'entre eux se rencontrent dans les milieux rocheux ( $21 \%$ exclusifs) et $72,3 \%$ dans les milieux herbacés $(4,8 \%$ exclusifs), alors que les taux de présence ne dépassent pas $25 \%$ dans les cinq autres catégories de milieux. Au total, 27,2\% des taxons endémiques sont exclusifs d'une des sept catégories principales de milieux. Ces résultats confirment qu'une forte proportion d'endémiques des Alpes est inféodée à des milieux rocheux ou d'éboulis (voir notamment PAWLOWSKI (1970: 212-213), OZENDA (1985: 62), Médail \& Verlaque (1997: 274), CasazZA \& al. (2005: 367), Новонм (2008), EssL \& al. (2009: 2552), RABITSCH \& Essl (2009: 895) et Noble \& Diadema (2011: 64)). Certaines interprétations précédemment énoncées au sujet des endémiques sont ainsi corroborées, comme à propos des plus faibles proportions de taxons adaptés à de forts changements d'humidité du sol et concernant une sténoécité pour le substrat plus élevée (AEsChIMANN \& al., 2012b: 195, 204).

De leur côté, les 502 taxons xénophytes sont particulièrement nitrophiles, puisque près de $95 \%$ d'entre eux sont présents dans ces milieux, souvent anthropiques, et que plus de $38 \%$ en sont même exclusifs, alors que les taux de présence ne dépassent pas $26,5 \%$ dans les six autres catégories de milieux. Cette prépondérance des milieux nitrophiles est aussi observée par DAINESE \& BRAgAZZA (2012) dans les Alpes italiennes. Au total, $41,8 \%$ des taxons xénophytes sont exclusifs d'une des sept catégories principales de milieux. La tendance méso-eutrophe des xénophytes mise en évidence précédemment (AESCHIMANN \& al., 2012b: 211) est ainsi étayée. En Corse, JEANMONOD \& al. (2011b: 92) ont obtenu des résultats comparables.

Tableau 1. - Pourcentages de taxons présents dans les sept catégories principales de milieux (caractères romains) et exclusifs de chaque catégorie (caractères italiques), calculés sur les nombres totaux de taxons indiqués entre crochets pour la flore totale et ses sous-ensembles. En gras le plus haut pourcentage de chaque colonne, les maximums absolus étant de plus encadrés (présents, exclusifs).

\begin{tabular}{|c|c|c|c|c|c|c|c|c|c|c|}
\hline \multirow{2}{*}{$\begin{array}{l}\text { Milieux } \\
\text { Aquatiques }\end{array}$} & \multicolumn{2}{|c|}{ Flore totale $[n=4485]$} & \multicolumn{2}{|c|}{ Endémiques [n=501] } & \multicolumn{2}{|c|}{ Indigènes [n=3482] } & \multicolumn{2}{|c|}{$\begin{array}{l}\text { Endémiques+indigènes } \\
{[\mathrm{n}=3983]}\end{array}$} & \multicolumn{2}{|c|}{ Xénophytes [n=502] } \\
\hline & 10,7 & 2,6 & 0,0 & 0,0 & 12,3 & 2,9 & 10,8 & 2,5 & 9,6 & 2,8 \\
\hline Nitrophiles & 57,0 & 8,5 & 21,2 & 0,6 & 56,7 & 5,4 & 52,2 & 4,8 & 94,8 & 38,2 \\
\hline Marécageux & 13,5 & 0,7 & 5,2 & 0,4 & 16,2 & 0,8 & 14,8 & 0,7 & 3,2 & 0,4 \\
\hline Herbacés & 64,9 & 2,5 & 72,3 & 4,8 & 70,0 & 2,5 & 70,3 & 2,8 & 22,3 & 0,2 \\
\hline
\end{tabular}


Le tableau 2 permet d'affiner les résultats, en listant les pourcentages de présence dans les 35 catégories secondaires de milieux. Plus de $30 \%$ des taxons indigènes non endémiques sont potentiellement présents dans trois catégories. Ainsi, dans les «pelouses, prés et pâturages secs, steppiques ou rocheux, du collinéen au subalpin moyen» (code «Flora alpina» 6.2), on dénombre $37,6 \%$ des taxons. Dans les «milieux rudéraux et reposoirs du bétail» (2.2 et 2.4) on en compte $34,1 \%$, et sur les «rochers s.1.» (3.2) on en recense encore $31,1 \%$. Trois autres catégories de milieux comptent plus de $25 \%$ d'indigènes. Ce sont les «éboulis s.1.» (3.3) avec 26,1\%, la «végétation arbustive des haies, lisières et buissons s.1.» (7.4) avec 28,1\% et les «forêts caducifoliées mésophiles» (9.2) avec 25,3\%.

Les valeurs du tableau 2 confirment le caractère nettement plus sténoèce des endémiques, car plus de $52 \%$ d'entre eux sont présents dans trois milieux seulement, alors que les proportions ne dépassent pas $25 \%$ dans les 32 autres milieux. Ainsi, sur les «rochers s.l.» (3.2), on recense $63,1 \%$ des taxons endémiques, le maximum absolu du tableau, de même que le plus haut pourcentage d'exclusifs, soit $8,4 \%$, ce qui représente 42 taxons. Parmi ces 42 endémiques exclusifs des rochers, quinze $(=36 \%)$ n'appartiennent qu'à deux genres; on compte en effet huit représentants du genre Moehringia (p. ex. M. intermedia, lebrunii et villosa) et sept du genre Saxifraga (p. ex. S. presolanensis, valdensis et vandellii). Les «éboulis s.1.» (3.3), hébergent 59,1\% des taxons endémiques, ainsi que 3,4\% d'exclusifs, ce qui représente 17 taxons (p. ex. Galium montis-arerae, Linaria tonzigii et Viola cenisia). Les «pelouses subalpines-alpines s.l.» (6.5) en hébergent 52,1\%, ainsi que 3,4\% d'exclusifs (soit 17 taxons, p. ex. Euphrasia christii, Gentiana engadinensis et Pulsatilla alpina subsp. schneebergensis). Parmi les 32 autres milieux, seuls les «pelouses, prés et pâturages secs, steppiques ou rocheux, du collinéen au subalpin moyen» (6.2) se distinguent avec $25 \%$ des endémiques, les proportions ne dépassant pas $15,8 \%$ dans les 31 autres milieux.

Le lien entre la présence d'endémiques et celle de milieux rocheux a été reconnu à plusieurs reprises, en particulier dans le monde méditerranéen (DAVIS, 1951; SNOGERUP, 1971; DANIN, 1999). D'une manière générale, les parois rocheuses sont considérées comme des refuges parce qu'elles présentent une stabilité dans le temps des principaux facteurs écologiques déterminants pour la présence des végétaux (rayonnement, température, eau, nutriments, compétition), tout en offrant une grande variabilité au niveau de ces facteurs sur des distances très faibles, principalement en fonction de la pente, de l'exposition et de la nature de la roche, en relation avec les facteurs d'altération (SCHROETER, 1926; DAVIS, 1951; SNOGERUP, 1971; DANIN, 1999; LARSON \& al., 2000). Il en résulte un affranchissement par rapport au climat général (THEURILLAT \& al., 1998; THEURILlAT \& GUISAN, 2001), permettant aux espèces capables de croître dans les fissures d'être moins affectées par les vicissitudes climatiques. C'est un aspect particulièrement important pour les endémiques, que ce soit pour le maintien à long terme des paléo-endémiques ou le développement des néo-endémiques. De la même manière, les éboulis constituent aussi des refuges stables dans le temps pour les plantes adaptées à un tel milieu (Schroeter, 1926; EllenberG, 1988; MÜLLER, 1997), lui aussi en bonne partie affranchi du climat général. En effet, la nature de l'éboulis, en particulier sa granulométrie et sa mouvance, est déterminante pour tout ce qui regarde les paramètres édaphiques et la compétition; la pente, l'exposition et la couleur du matériel interviennent de leur côté au niveau de la température. Les pelouses subalpinesalpines, troisième milieu le plus important du point de vue de la présence des endémiques, montrent aussi une stabilité dans le temps, du moins à l'étage alpin. Ainsi, selon TinNer \& THEurillat (2003), les pelouses alpines sur silice dans la région d'Aletsch (Alpes suisses) existent sans interruption depuis au moins 11'000 ans, alors que STEINGER \& al. (1996) ont estimé à 2000 ans l'âge d'un clone de Carex curvula. Par ailleurs, l'abaissement de la limite supérieure de la forêt par l'homme a offert aux espèces alpines une possibilité d'extension dans l'étage subalpin depuis plusieurs millénaires (WIERSEMA, 1989; THeuRILlat \& al., 1998). Enfin, si les pelouses sèches sont en majeure partie des milieux de substitution dans les Alpes, il existe néanmoins des surfaces réduites naturelles, relativement stables, dans les complexes de milieux qui se rencontrent au contact des parois rocheuses, des affleurements rocheux et des éboulis. Les espèces de pelouses pouvaient alors y persister à l'abri de la concurrence des arbres et des buissons (ElLENBERG, 1988), permettant par là même le maintien d'endémiques qui ont pu s'étendre secondairement.

Le caractère particulièrement nitrophile des xénophytes est confirmé par le tableau 2. En effet, $62,2 \%$ d'entre eux sont recensés dans les «milieux rudéraux et reposoirs du bétail» (codes «Flora alpina» 2.2 et 2.4), ainsi que $35,9 \%$ dans les «cultures et friches» (2.1). Ces résultats corroborent ceux de WALTER \& al. en Autriche (2005: 15). De plus, 62,7\% des xénophytes peuvent être cultivés (2.9) et 4,4\% le sont exclusivement. Parmi les 32 autres milieux, il est intéressant de remarquer que plus de $20 \%$ des xénophytes se rencontrent dans la «végétation arbustive des haies, lisières et buissons s.1.» (7.4: 23,3\% des taxons), ainsi que sur les «berges, alluvions, etc.» (2.5: 20,7\%). Avec 67 taxons comptant dans la catégorie des «rochers s.l.» (3.2), soit 13,3\%, les xénophytes pourraient représenter une menace pour la flore endémique de ces milieux, surtout dans un contexte de réchauffement climatique de 2 à $5^{\circ} \mathrm{C}$ (MorADI \& al., 2012) qui leur permettrait d'entrer en contact avec les taxons occupant actuellement les étages montagnard et subalpin (DAINESE \& BRAGAZZA, 2012: 18), parmi lesquels se trouvent de nombreux endémiques (AESCHIMANN \& al., 2011b: 236-237). Les proportions assez élevées de xénophytes dans la végétation arbustive (7.4) et forestière caducifoliée (9.2) est à mettre en relation avec la naturalisation 
Tableau 2. - Pourcentages de taxons présents dans les 35 catégories secondaires de milieux (caractères romains) et exclusifs de chaque catégorie (caractères italiques), calculés sur les nombres totaux de taxons indiqués entre crochets pour la flore totale et ses sous-ensembles. Pour les taxons exclusifs, seules les valeurs $\geq 1 \%$ sont indiquées. En gras les pourcentages de taxons présents $>30 \%$, les maximums absolus étant de plus encadrés (présents, exclusifs).

\begin{tabular}{|c|c|c|c|c|c|c|c|c|c|c|c|}
\hline \multirow{2}{*}{$\begin{array}{c}\begin{array}{c}\text { Codes } \\
\text { «Flora } \\
\text { alpina» }\end{array} \\
1.1-1.3\end{array}$} & \multirow{2}{*}{$\begin{array}{l}\text { Milieux } \\
\text { Milieux aquatiques permanents }\end{array}$} & \multicolumn{2}{|c|}{$\begin{array}{l}\text { Flore totale } \\
{[n=4485]}\end{array}$} & \multicolumn{2}{|c|}{$\begin{array}{l}\text { Endémiques } \\
{[\mathrm{n}=501]}\end{array}$} & \multicolumn{2}{|c|}{$\begin{array}{l}\text { Indigènes } \\
\text { [n=3482] }\end{array}$} & \multicolumn{2}{|c|}{$\begin{array}{l}\text { Endémiques } \\
\text { +indigènes } \\
\text { [n=3983] }\end{array}$} & \multicolumn{2}{|c|}{$\begin{array}{c}\text { Xénophytes } \\
{[n=502]}\end{array}$} \\
\hline & & 6,0 & 2,1 & 0,0 & & 7,1 & 2,3 & 6,2 & 2,0 & 4,6 & 2,4 \\
\hline 2.1 & Cultures et friches & 22,4 & & 1,6 & & 23,5 & & 20,7 & & 35,9 & \\
\hline $2.2 ; 2.4$ & Milieux rudéraux et reposoirs du bétail & 34,0 & 1,0 & 5,4 & & 34,1 & & 30,5 & & 62,2 & 4,0 \\
\hline $2.7-2.8$ & Jardins, vergers, etc. & 6,0 & & 0,0 & & 5,7 & & 5,0 & & 13,9 & \\
\hline 2.9 & Cultivé & 11,1 & & 1,2 & & 5,1 & & 4,6 & & 62,7 & 4,4 \\
\hline $3.1 ; 3.5$ & Milieux graveleux ou sablonneux & 10,3 & & 4,8 & & 11,7 & & 10,8 & & 6,0 & \\
\hline 3.2 & Rochers s.l. & 32,7 & 1,7 & 63,1 & 8,4 & 31,1 & & 35,1 & 1,9 & 13,3 & \\
\hline 3.3 & Eboulis s.l. & 27,5 & & 59,1 & 3,4 & 26,1 & & 30,2 & & 5,6 & \\
\hline 5.2 & Tourbières & 2,8 & & 0,0 & & 3,5 & & 3,1 & & 0,8 & \\
\hline 6.1 & Pelouses xérophiles méditerranéennes & 2,6 & & 0,0 & & 3,1 & & 2,7 & & 1,6 & \\
\hline 6.2 & $\begin{array}{l}\text { Pelouses, prés et pâturages secs, } \\
\text { steppiques ou rocheux, du collinéen } \\
\text { au subalpin moyen }\end{array}$ & 32,9 & & 25,0 & & 37,6 & & 36,0 & & 8,8 & \\
\hline 6.3 & Prés et pâturages mésophiles et hygrophiles & 19,5 & & 7,2 & & 22,7 & & 20,7 & & 9,8 & \\
\hline 6.4 & $\begin{array}{l}\text { Prés, pâturages et pelouses maigres, } \\
\pm \text { humides et acidophiles }\end{array}$ & 1,7 & & 0,2 & & 2,1 & & 1,9 & & 0,0 & \\
\hline 6.5 & Pelouses subalpines-alpines s.l. & 21,4 & & 52,1 & 3,4 & 20,1 & & 24,1 & & 0,2 & \\
\hline 6.6 & Combes à neige & 2,4 & & 5,2 & & 2,4 & & 2,7 & & 0,0 & \\
\hline $6.7 ; 2.3$ & $\begin{array}{l}\text { Végétation herbacée des lisières } \\
\text { méso-thermophiles et des lisières } \\
\text { fraîches nitrophiles }\end{array}$ & 19,2 & & 7,2 & & 22,5 & & 20,6 & & 8,8 & \\
\hline 7.5 & Saulaies riveraines basses & 1,6 & & 0,4 & & 1,8 & & 1,7 & & 1,6 & \\
\hline 7.6 & Maquis et garrigues & 1,4 & & 0,0 & & 1,6 & & 1,4 & & 2,0 & \\
\hline 8.1 & Aulnaies vertes et saulaies subalpines & 3,3 & & 3,8 & & 3,6 & & 3,7 & & 0,0 & \\
\hline 8.2 & Brousses de pins de montagne & 4,3 & & 5,2 & & 4,8 & & 4,9 & & 0,0 & \\
\hline 9.1 & Forêts de conifères & 19,4 & & 15,8 & & 22,6 & & 21,7 & & 0,8 & \\
\hline 9.2 & Forêts caducifoliées mésophiles & 22,2 & & 5,8 & & 25,3 & & 22,9 & & 16,7 & \\
\hline 9.3 & Chênaies et ostryaies thermophiles & 10,8 & & 3,2 & & 13,0 & & 11,8 & & 3,0 & \\
\hline 9.4 & Chênaies sempervirentes & 1,3 & & 0,0 & & 1,5 & & 1,3 & & 1,2 & \\
\hline 9.5 & Populations de Robinia pseudoacacia & 0,7 & & 0,0 & & 0,8 & & 0,7 & & 1,2 & \\
\hline
\end{tabular}


des espèces ornementales échappées de jardins, grâce au réchauffement climatique en cours («laurophyllisation»: CARRARO \& al., 2001; WALTHER, 1999) et de plus facilitée par les dépôts de déchets organiques en forêt (RUSTERHOLz \& al., 2012). L'importante proportion de xénophytes sur les berges et les alluvions (2.5) intervient surtout à l'étage collinéen (SINISCALCO \& al., 2011; BARNI \& al., 2012; DAINESE \& BRAGAZZA, 2012). En effet, le nombre de xénophytes diminue rapidement avec l'altitude (AESCHIMANN \& al., 2011b: 237), ce que corrobore l'absence de ce groupe dans les «pelouses subalpinesalpines» (6.5), dans les «combes à neige» (6.6), dans les «aulnaies vertes et saulaies subalpines» (8.1) et dans les «brousses de pins de montagne» (8.2), en confirmation des observations de BECKER \& al. (2005) dans les Alpes suisses, de Walter \& al. (2005) en Autriche et de Dainese \& Bragazza (2012) dans les Alpes italiennes, étendues au niveau intercontinental par SEIPEL \& al. (2012).

L'histogramme de la figure 1 caractérise l'amplitude écologique de chacun des trois groupes de taxons: endémiques, indigènes (non endémiques) et xénophytes. Ici encore, la sténoécité marquée des endémiques est mise en évidence, puisque parmi ces 501 taxons, 17\% (85 taxons) se rencontrent dans une seule des 35 catégories secondaires de milieux, alors que seulement $7,4 \%$ des indigènes répondent à ce critère. A l'inverse, $11,8 \%$ des indigènes sont présents dans six milieux, mais seulement $6 \%$ des endémiques. Un cumul des pourcentages pour un à trois milieux occupés donne $62,5 \%$ pour les endémiques (313 taxons) contre 38,7\% pour les indigènes. La forte tendance sténoèce des endémiques est donc aussi bien avérée en ce qui concerne les milieux que pour ce qui est du substrat préféré et des valeurs indicatrices du sol (AESCHIMANN \& al., 2012b: 195, 204, 211). Parmi les 502 xénophytes, 12,7\% se rencontrent dans une seule des 35 catégories secondaires de milieux, un résultat intermédiaire entre ceux des endémiques et des indigènes. Pour deux à trois milieux occupés, ce sont en revanche les pourcentages de xénophytes qui sont les plus élevés, respectivement $25,3 \%$ et $27,3 \%$. Le cumul des pourcentages pour un à trois milieux occupés est de $65,3 \%$ pour les xénophytes, un résultat en accord avec le fait que les taxons de ce groupe se concentrent dans les milieux nitrophiles (voir les tableaux 1 et 2). Le caractère très euryèce des xénophytes, démontré surtout pour le substrat préféré et dans une moindre mesure pour les valeurs indicatrices du sol (AESCHIMANN \& al., 2012b) ne se retrouve donc pas en ce qui concerne les milieux.

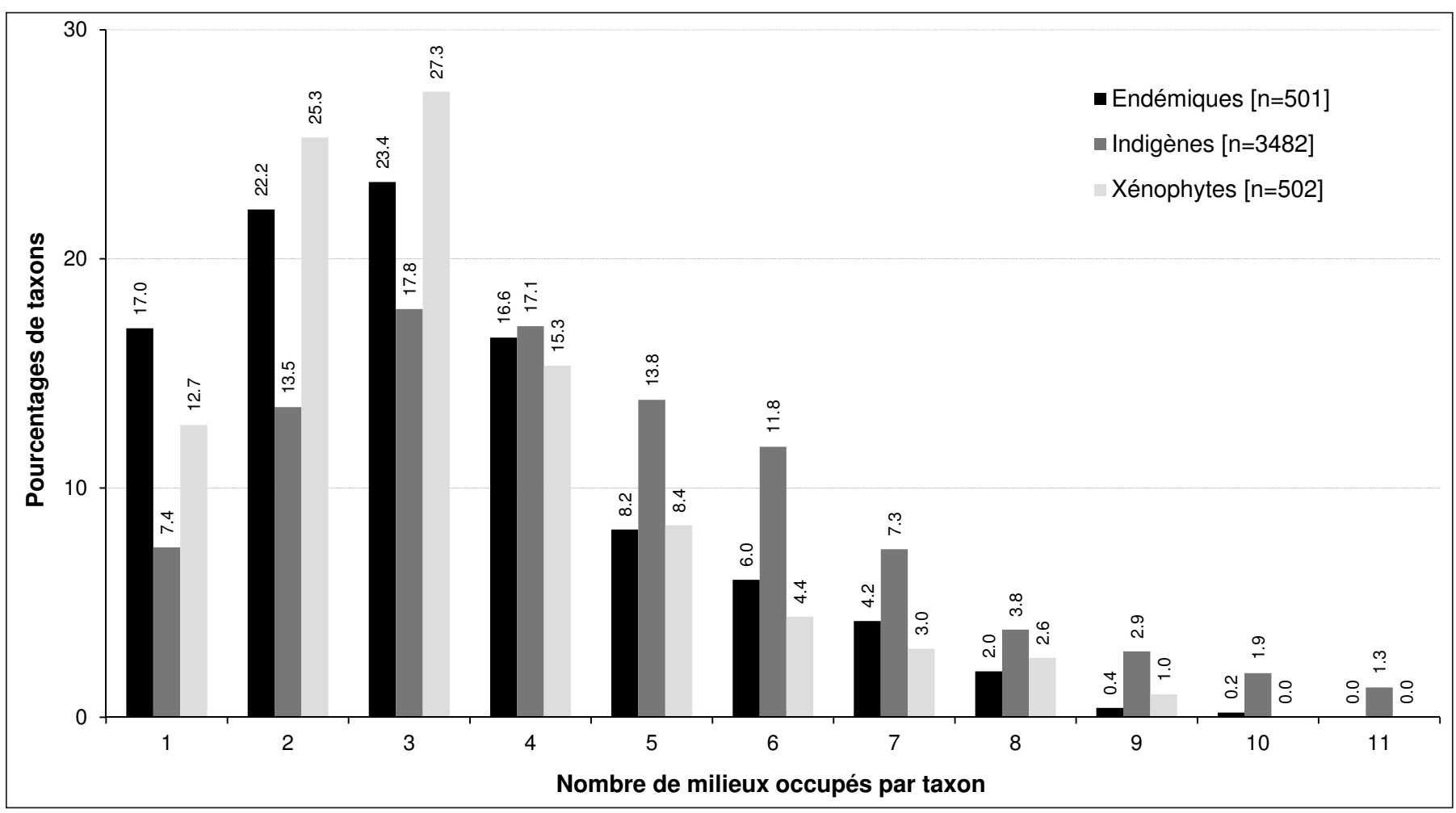

Fig. 1. - Pourcentages de taxons par nombre de milieux occupés, calculés sur les nombres totaux de taxons indiqués entre crochets pour les endémiques, les indigènes non endémiques et les xénophytes. Les 35 catégories secondaires de milieux sont ici considérées et les pourcentages $>1 \%$ pour les indigènes déterminent le nombre de colonnes. 


\section{Etages de végétation}

Les proportions par étage de végétation de taxons recensés dans les sept catégories principales de milieux sont indiquées dans le tableau 3 , où ressort un gradient altitudinal pour chaque catégorie. Ainsi, de l'étage collinéen à l'étage nival, les proportions de taxons comptant dans les milieux aquatiques et nitrophiles diminuent avec l'altitude. A l'inverse, la proportion de taxons présents dans les milieux rocheux augmente très fortement de l'étage collinéen à l'étage nival. En revanche, les pourcentages dans les milieux marécageux et herbacés montrent une augmentation de l'étage collinéen à l'étage alpin, suivie d'une diminution à l'étage nival. Les distributions des milieux buissonneux et forestiers présentent chacune un mode à l'étage montagnard.

A l'étage collinéen, c'est dans les milieux nitrophiles qu'on relève le pourcentage le plus élevé de taxons avec $65,5 \%$, suivis par les milieux herbacés avec $61,2 \%$. L'ordre s'inverse à l'étage montagnard, avec la dominance des taxons des milieux herbacés, soit $68,4 \%$, contre $57,8 \%$ pour les milieux nitrophiles. Le pourcentage de taxons dans les milieux herbacés atteint $75,1 \%$ à l'étage subalpin et $81,7 \%$ à l'étage alpin, où une très forte proportion de taxons se rencontre aussi dans les milieux rocheux, soit $74,1 \%$. L'étage nival se distingue avec $96,7 \%$ de taxons présents dans les milieux rocheux, le maximum absolu du tableau.

Apparemment paradoxaux, les pourcentages non négligeables de taxons des milieux buissonneux et forestiers relevés aux étages alpin et nival s'expliquent par la présence à ces altitudes élevées de taxons qui se rencontrent dans ces deux milieux à des altitudes inférieures. Ainsi, Luzula lutea est recensée jusqu'à l'étage nival, mais sa présence dans les landes aux étages subalpin et alpin inférieur fait qu'elle compte dans les milieux buissonneux, d'où la mention de cette catégorie aussi à l'étage nival. Il en va de même pour Homogyne alpina, espèce des forêts et des landes de l'étage collinéen au subalpin, qui se rencontre dans les pelouses alpines et atteint l'étage nival.
Les variations de proportions dans les milieux aquatiques, marécageux et nitrophiles corroborent les résultats précédemment développés à propos des valeurs indicatrices écologiques (humidité et niveau trophique du sol); voir AESCHIMANN \& al. (2012b: 196-197, 211-212), aussi pour les diverses interprétations à transposer ici. De même, l'augmentation de la proportion de taxons des milieux rocheux va de pair avec l'augmentation des fortes pentes avec l'altitude calculée pour la Suisse (Theurillat \& Guisan, 2001).

Le tableau 4 permet d'affiner les résultats, en listant les pourcentages de présence dans les 35 catégories secondaires de milieux. Comme pour les catégories principales (tableau 3 ), il ressort un gradient altitudinal pour presque tous les milieux. Pour certains, le gradient est le même que celui de la catégorie principale, par exemple pour les «cultures et friches» (code «Flora alpina» 2.1) et les «milieux rudéraux et reposoirs du bétail» (2.2 et 2.4), dont le pourcentage de taxons diminue avec l'altitude; ou pour les «rochers s.1.» (3.2) et les «éboulis s.1.» (3.3), dont le pourcentage augmente avec l'altitude. En revanche, certaines catégories secondaires de milieux montrent un gradient différent. Par exemple, pour les «pelouses, prés et pâturages secs» (6.2), le pourcentage diminue avec l'altitude.

A l'étage collinéen, trois milieux rassemblent chacun plus de $30 \%$ des taxons. Les «milieux rudéraux et reposoirs du bétail» (2.2 et 2.4) sont au premier rang, avec $40,9 \%$ des taxons, suivis des «pelouses, prés et pâturages secs, steppiques ou rocheux, du collinéen au subalpin moyen» (6.2) et de la «végétation arbustive des haies, lisières et buissons s.1.» (7.4). On dénombre aussi plus de $25 \%$ des taxons dans les «cultures et friches» (2.1), sur les «rochers s.1.» (3.2) et dans les «forêts caducifoliées mésophiles» (9.2). A l'étage montagnard, trois milieux également rassemblent chacun plus de $30 \%$ des taxons. Les «pelouses, prés et pâturages secs, steppiques ou rocheux, du collinéen au subalpin moyen» (6.2) passent au premier rang, avec $37,2 \%$ des taxons, suivis des «milieux rudéraux et reposoirs du bétail» (2.2 et 2.4) et des «rochers s.1.» (3.2). On dénombre aussi plus de $25 \%$ des taxons dans la «végétation

Tableau 3. - Pourcentages de taxons potentiellement présents dans les sept catégories principales de milieux, calculés sur les nombres totaux de taxons indiqués entre crochets pour chaque étage de végétation, ainsi que pour la flore totale. En gras le plus haut pourcentage de chaque colonne, le maximum absolu étant de plus encadré.

\begin{tabular}{lccccc|c}
\hline Milieux & Collinéen [n=3515] & Montagnard [n=3325] & Subalpin [n=2400] & Alpin [n=1099] & Nival [n=152] & Flore totale [n=4485] \\
\hline Aquatiques & 13,2 & 10,0 & 6,7 & 2,8 & 0,0 & 10,7 \\
Nitrophiles & 65,5 & 57,8 & 48,2 & 33,8 & 31,6 & 57,0 \\
Rocheux & 38,7 & 46,1 & 57,8 & 74,1 & 96,7 & 45,9 \\
Marécageux & 12,9 & 16,2 & 18,8 & 20,3 & 11,8 & 13,5 \\
Herbacés & 61,2 & 68,4 & 75,1 & 81,7 & 77,6 & 64,9 \\
Buissonneux & 42,8 & 44,7 & 43,4 & 35,9 & 21,1 & 6,6 \\
Forestiers & 41,2 & 44,3 & 40,4 & 27,3 & 30,6 & 36,9 \\
\hline
\end{tabular}


Tableau 4. - Pourcentages de taxons potentiellement présents dans les 35 catégories secondaires de milieux, calculés sur les nombres totaux de taxons indiqués entre crochets pour chaque étage de végétation, ainsi que pour la flore totale. En gras les pourcentages $>30 \%$, le maximum absolu étant de plus encadré.

\begin{tabular}{|c|c|c|c|c|c|c|c|}
\hline $\begin{array}{l}\text { Codes } \\
\text { «Flora } \\
\text { alpina» }\end{array}$ & Milieux & $\begin{array}{l}\text { Collinéen } \\
\text { [n=3515] }\end{array}$ & $\begin{array}{l}\text { Montagnard } \\
{[\mathrm{n}=3325]}\end{array}$ & $\begin{array}{l}\text { Subalpin } \\
\text { [n=2400] }\end{array}$ & $\begin{array}{c}\text { Alpin } \\
{[n=1099]}\end{array}$ & $\begin{array}{c}\text { Nival } \\
{[n=152]}\end{array}$ & $\begin{array}{c}\text { Flore totale } \\
{[\mathrm{n}=4485]}\end{array}$ \\
\hline 1.1-1.3 & Milieux aquatiques permanents & 7,5 & 5,8 & 3,7 & 1,5 & 0,0 & 6,0 \\
\hline 1.4 & Milieux aquatiques temporaires & 7,6 & 5,7 & 3,8 & 1,5 & 0,0 & 6,1 \\
\hline 2.1 & Cultures et friches & 28,4 & 22,1 & 15,3 & 4,1 & 0,0 & 22,4 \\
\hline $2.2 ; 2.4$ & Milieux rudéraux et reposoirs du bétail & 40,9 & 34,0 & 26,3 & 14,6 & 9,9 & 34,0 \\
\hline 2.5 & Berges, alluvions, etc. & 19,8 & 19,8 & 20,8 & 18,8 & 28,3 & 18,4 \\
\hline 2.6 & Coupes forestières, etc. & 15,3 & 17,3 & 16,5 & 8,7 & 0,0 & 13,3 \\
\hline $2.7-2.8$ & Jardins, vergers, etc. & 7,6 & 6,7 & 5,5 & 2,2 & 0,0 & 6,0 \\
\hline 2.9 & Cultivé & 13,8 & 9,3 & 4,8 & 1,5 & 0,0 & 11,1 \\
\hline $3.1 ; 3.5$ & Milieux graveleux ou sablonneux & 11,8 & 11,5 & 10,5 & 8,3 & 7,9 & 10,3 \\
\hline 3.2 & Rochers s.l. & 27,4 & 32,9 & 41,3 & 53,7 & 86,2 & 32,7 \\
\hline 3.3 & Eboulis s.l. & 18,9 & 27,6 & 42,0 & 61,3 & 89,5 & 27,5 \\
\hline 3.4 & Lapiez & 2,0 & 3,5 & 5,8 & 9,8 & 9,2 & 3,1 \\
\hline 4.1-4.2 & Sources et ruisseaux & 6,9 & 9,5 & 11,8 & 14,5 & 11,2 & 8,0 \\
\hline 5.1 & Bas-marais et marais de transition & 8,0 & 9,7 & 11,6 & 13,1 & 7,2 & 8,1 \\
\hline 5.2 & Tourbières & 3,0 & 3,7 & 3,9 & 3,5 & 0,7 & 2,8 \\
\hline 6.1 & Pelouses xérophiles méditerranéennes & 3,3 & 2,1 & 0,6 & 0,2 & 0,0 & 2,6 \\
\hline 6.2 & $\begin{array}{l}\text { Pelouses, prés et pâturages secs, steppiques } \\
\text { ou rocheux, du collinéen au subalpin moyen }\end{array}$ & 38,1 & 37,2 & 31,1 & 21,3 & 5,3 & 32,9 \\
\hline 6.3 & Prés et pâturages mésophiles et hygrophiles & 20,6 & 23,4 & 24,1 & 21,5 & 3,9 & 19,5 \\
\hline 6.4 & $\begin{array}{l}\text { Prés, pâturages et pelouses maigres, } \\
\pm \text { humides et acidophiles }\end{array}$ & 1,7 & 2,2 & 2,8 & 3,5 & 1,3 & 1,7 \\
\hline 6.5 & Pelouses subalpines-alpines s.l. & 10,1 & 21,0 & 39,2 & 67,9 & 71,7 & 21,4 \\
\hline 6.6 & Combes à neige & 0,2 & 1,6 & 4,3 & 9,5 & 24,3 & 2,4 \\
\hline $6.7 ; 2.3$ & $\begin{array}{l}\text { Végétation herbacée des lisières méso-thermophiles } \\
\text { et des lisières fraîches nitrophiles }\end{array}$ & 22,8 & 23,1 & 19,8 & 8,4 & 0,7 & 19,2 \\
\hline 7.1 & Landes s.l. & 6,8 & 11,0 & 16,2 & 24,6 & 17,8 & 9,5 \\
\hline 7.2 & Mégaphorbiées s.l. & 8,1 & 11,7 & 14,8 & 14,7 & 2,0 & 9,4 \\
\hline 7.3 & Garrigues basses et bas-maquis & 8,1 & 5,4 & 2,7 & 0,7 & 0,0 & 6,4 \\
\hline 7.4 & $\begin{array}{l}\text { Végétation arbustive des haies, } \\
\text { lisières et buissons s.l. }\end{array}$ & 30,7 & 29,5 & 24,3 & 8,1 & 0,0 & 25,6 \\
\hline 7.5 & Saulaies riveraines basses & 1,9 & 1,9 & 2,0 & 1,4 & 0,0 & 1,6 \\
\hline 7.6 & Maquis et garrigues & 1,8 & 0,7 & 0,1 & 0,0 & 0,0 & 1,4 \\
\hline 8.1 & Aulnaies vertes et saulaies subalpines & 2,1 & 3,9 & 6,0 & 7,4 & 3,3 & 3,3 \\
\hline 8.2 & Brousses de pins de montagne & 3,0 & 5,6 & 8,0 & 10,4 & 3,3 & 4,3 \\
\hline 9.1 & Forêts de conifères & 19,7 & 25,1 & 27,8 & 23,8 & 6,6 & 19,4 \\
\hline 9.2 & Forêts caducifoliées mésophiles & 26,4 & 27,0 & 23,5 & 11,2 & 0,7 & 22,2 \\
\hline 9.3 & Chênaies et ostryaies thermophiles & 13,5 & 12,8 & 9,0 & 3,1 & 0,7 & 10,8 \\
\hline 9.4 & Chênaies sempervirentes & 1,7 & 1,0 & 0,4 & 0,2 & 0,0 & 1,3 \\
\hline 9.5 & Populations de Robinia pseudoacacia & 0,9 & 0,8 & 0,7 & 0,1 & 0,0 & 0,7 \\
\hline
\end{tabular}


arbustive des haies, lisières et buissons s.1.» (7.4), dans les «éboulis s.1.» (3.3), dans les «forêts caducifoliées mésophiles» (9.2) et dans les «forêts de conifères» (9.1). A l'étage subalpin, ce sont quatre milieux qui rassemblent chacun plus de $30 \%$ des taxons. Les «éboulis s.l.» (3.3) passent au premier rang, avec $42 \%$ des taxons, suivis des «rochers s.1.» (3.2), des «pelouses subalpines-alpines s.1.» (6.5) et des «pelouses, prés et pâturages secs, steppiques ou rocheux, du collinéen au subalpin moyen» (6.2). On dénombre aussi plus de $25 \%$ des taxons dans les «forêts de conifères» (9.1) et dans les «milieux rudéraux et reposoirs du bétail» (2.2 et 2.4). A l'étage alpin, trois milieux rassemblent chacun plus de $50 \%$ des taxons et aucun autre ne se distingue avec plus de $25 \%$ des taxons. Les «pelouses subalpines-alpines s.1.» (6.5), passent au premier rang, avec 67,9\% des taxons, suivies des «éboulis s.l.» (3.3) et des «rochers s.1.» (3.2). A l'étage nival, trois milieux rassemblent chacun plus de $70 \%$ des taxons. Les «éboulis s.l.» (3.3) repassent au premier rang, avec $89,5 \%$ des taxons (le maximum absolu du tableau), suivis des «rochers s.1.» (3.2) et des «pelouses subalpines-alpines s.1.» (6.5). Parmi les 32 autres milieux, un seul se distingue avec plus de $25 \%$ des taxons, les «berges, alluvions, etc.» (2.5).

Comme le tableau 3 , le tableau 4 comporte quelques pourcentages apparemment paradoxaux, et ce pour les mêmes raisons. Ainsi, $0,2 \%$ pour les «combes à neige» (6.6) à l'étage collinéen peut intriguer, mais ce pourcentage s'explique aisément lorsqu'on voit quels taxons sont concernés, comme Poa alpina, présent dans les combes à neige en altitude, mais signalé parfois jusqu'en plaine, ou bien à l'inverse Plantago lanceolata, fréquent en plaine, mais signalé occasionnellement jusque dans les combes à neige en altitude. Et l'on retrouve à nouveau des pourcentages apparemment paradoxaux pour les étages alpin et nival dans les milieux 8.1 à 9.5. Les interprétations et quelques exemples cités plus haut à propos du tableau 3 sont valables ici aussi. Le $0,7 \%$ à l'étage nival aussi bien pour les «forêts caducifoliées mésophiles» (9.2) que pour les «chênaies et ostryaies thermophiles» (9.3) est le fait de
Sesleria caerulea, dont la très large amplitude altitudinale et la grande tolérance écologique font qu'on trouve cette Graminée aussi bien à l'étage nival qu'en basse altitude dans les milieux 9.2 et 9.3 .

\section{Contingents floristiques}

Le tableau 5 exprime les pourcentages de taxons présents dans les sept catégories principales de milieux, pour les quatre principaux contingents floristiques mis en évidence précédemment (AEsCHIMANN \& al., 2011b: 244), ainsi que pour le cortège floristique arctico-alpin qui occupe le second rang en importance aux étages alpin et nival. Les profils des contingents européen et eurasiatique sont ceux qui se rapprochent le plus de celui de la flore totale, avec toutefois des proportions plus élevées dans les milieux nitrophiles, buissonneux et forestiers, mais plus basses dans les milieux rocheux, des résultats en accord avec la faible représentation de ces deux contingents floristiques dans les étages supérieurs (AEschimanN \& al., 2011b: 246). Le contingent européen se distingue par de fortes proportions dans les milieux herbacés $(70,6 \%)$, forestiers $(56,8 \%)$ et marécageux $(17,1 \%)$. Parmi les taxons du contingent eurasiatique, $73,1 \%$ sont présents dans les milieux nitrophiles et $18,1 \%$ dans les milieux aquatiques. Pour l'interprétation de ce dernier pourcentage, relativement élevé, voir AESCHIMANN $\&$ al. (2012a: 11, 19; 2012b: 201). Le cortège floristique méditerranéen montre une proportion élevée de taxons présents dans les milieux nitrophiles, soit $77,7 \%$, les taxons de ce cortège étant en effet nombreux dans les cultures, les friches et les milieux rudéraux. A l'inverse, les proportions de taxons méditerranéens sont faibles dans les milieux forestiers $(19,3 \%)$ et marécageux $(1,5 \%)$. Le profil du cortège floristique sudeuropéen-montagnard, dominant à l'étage alpin (AESCHIMANN \& al., 2011b: 246), est comparable à celui de cet étage (tableau 3). Dans le cortège floristique arctico-alpin, les pourcentages élevés de taxons présents dans les milieux herbacés $(80,8 \%)$, rocheux $(65,4 \%)$ et marécageux $(40,4 \%)$ sont à l'image de la végétation du type toundra.

Tableau 5. - Pourcentages de taxons présents dans les sept catégories principales de milieux, calculés sur les nombres totaux de taxons indiqués entre crochets pour cinq contingents floristiques sélectionnés, ainsi que pour la flore totale. En gras le plus haut pourcentage de chaque colonne, le maximum absolu étant de plus encadré.

\begin{tabular}{lccccc|c}
\hline Milieux & S-Eur.-Mont. [n=1262] & Médit. [n=779] & Eur. [n=759] & Eurasiat. [n=631] & Arct.-Alp. [n=104] & Flore totale [n=4485] \\
\hline Aquatiques & 0,3 & 5,1 & 11,6 & 18,1 & 6,7 & 10,7 \\
Nitrophiles & 29,2 & $\mathbf{7 7 , 7}$ & 63,0 & $\mathbf{7 3 , 1}$ & 45,2 & 57,0 \\
Rocheux & 76,4 & 43,1 & 32,8 & 31,5 & 65,4 & 45,9 \\
Marécageux & 10,1 & 1,5 & 17,1 & 13,3 & 40,4 & 13,5 \\
Herbacés & $\mathbf{7 8 , 1}$ & 63,3 & $\mathbf{7 0 , 6}$ & 63,2 & $\mathbf{8 0 , 8}$ \\
Buissonneux & 37,1 & 41,2 & 48,1 & 48,3 & 29,8 & 13,5 \\
Forestiers & 31,6 & 19,3 & 56,8 & 51,2 & 30,6 & 36,9 \\
\hline
\end{tabular}




\section{Familles}

Parmi les douze familles réunissant chacune plus de 100 taxons dans les Alpes (AEschimAnN \& al., 2011a: 39), celle des Cyperaceae montre nettement les proportions les plus élevées de taxons présents dans les milieux marécageux et aquatiques (tableau 6), soit respectivement 53,8\% et $41,3 \%$. Dans les milieux herbacés et nitrophiles, toutes les familles affichent des proportions relativement élevées ( $>34 \%$ ). Les Poaceae ont le pourcentage le plus élevé dans les milieux nitrophiles, soit 74,7\%, mais comptent aussi $74,9 \%$ de taxons dans les milieux herbacés. Ce sont les Fabaceae qui montrent le plus haut pourcentage dans les milieux herbacés, soit $81,4 \%$, le maximum absolu du tableau, et en revanche le plus faible pourcentage dans les milieux marécageux, soit $3,6 \%$, des résultats en accord avec ceux relatifs aux valeurs indicatrices d'humidité du sol, comme c'est aussi le cas pour les Cyperaceae (AESCHIMANN \& al., 2012b: 201). La plus forte proportion de taxons présents dans les milieux rocheux est celle des Caryophyllaceae, soit 62,4\%. En effet, cette famille compte 21,6\% d'endémiques (AESCHIMANN \& al., 2011a: 4647). On ne compte à l'inverse que $17,5 \%$ de taxons présents dans les milieux rocheux parmi les Cyperaceae, le plus bas pourcentage pour ces milieux. Ce sont les Rosaceae qui ont les

Tableau 6. - Pourcentages de taxons présents dans les sept catégories principales de milieux, calculés sur les nombres totaux de taxons recensés dans les douze familles réunissant chacune plus de 100 taxons dans les Alpes, ainsi que pour la flore totale. En gras le plus haut pourcentage de chaque colonne, le maximum absolu étant de plus encadré.

\begin{tabular}{|c|c|c|c|c|c|c|c|c|}
\hline Famille & Total taxons & Aquatiques & Nitrophiles & Rocheux & Marécageux & Herbacés & Buissonneux & Forestiers \\
\hline Asteraceae & 557 & 8,4 & 71,1 & 50,8 & 14,9 & 79,4 & 47,6 & 39,5 \\
\hline Poaceae & 359 & 9,5 & 74,7 & 54,3 & 11,7 & 74,9 & 32,6 & 29,5 \\
\hline Fabaceae & 280 & 4,3 & 63,6 & 42,9 & 3,6 & 81,4 & 49,3 & 40,4 \\
\hline Caryophyllaceae & 210 & 4,3 & 44,3 & 62,4 & 7,6 & 67,1 & 17,1 & 18,6 \\
\hline Scrophulariaceae & 190 & 7,9 & 62,1 & 43,7 & 15,3 & 76,3 & 31,6 & 28,9 \\
\hline Cyperaceae & 160 & 41,3 & 40,6 & 17,5 & 53,8 & 66,3 & 25,6 & 33,1 \\
\hline Ranunculaceae & 144 & 14,6 & 34,7 & 29,2 & 13,9 & 75,7 & 41,7 & 51,4 \\
\hline Lamiaceae & 138 & 10,9 & 73,9 & 51,4 & 8,7 & 79,0 & 50,0 & 42,8 \\
\hline Liliaceae & 109 & 2,8 & 67,9 & 47,7 & 7,3 & 80,7 & 64,2 & 48,6 \\
\hline Flore totale & 4485 & 10,7 & 57,0 & 45,9 & 13,5 & 64,9 & 40,6 & 36,9 \\
\hline
\end{tabular}

Tableau 7. - Pourcentages de taxons présents dans les sept catégories principales de milieux, calculés sur les nombres totaux de taxons recensés dans les quinze genres réunissant chacun au moins 40 taxons dans les Alpes, ainsi que pour la flore totale. En gras le plus haut pourcentage de chaque colonne, le maximum absolu étant de plus encadré.

\begin{tabular}{|c|c|c|c|c|c|c|c|c|c|}
\hline Genre & Famille & Total taxons & Aquatiques & Nitrophiles & Rocheux & Marécageux & Herbacés & Buissonneux & Forestiers \\
\hline Carex & Cyperaceae & 115 & 30,4 & 44,3 & 22,6 & 54,8 & 75,7 & 33,0 & 43,5 \\
\hline Festuca & Poaceae & 70 & 1,4 & 48,6 & 78,6 & 11,4 & 92,9 & 32,9 & 35,7 \\
\hline Saxifraga & Saxifragaceae & 59 & 0,0 & 13,6 & 98,3 & 11,9 & 49,2 & 10,2 & 8,5 \\
\hline Campanula & Campanulaceae & 51 & 0,0 & 45,1 & 62,7 & 2,0 & 76,5 & 43,1 & 41,2 \\
\hline Galium & Rubiaceae & 51 & 7,8 & 49,0 & 47,1 & 9,8 & 74,5 & 47,1 & 49,0 \\
\hline Euphorbia & Euphorbiaceae & 43 & 11,6 & 67,4 & 34,9 & 0,0 & 79,1 & 44,2 & 39,5 \\
\hline Viola & Violaceae & 42 & 4,8 & 33,3 & 42,9 & 16,7 & 85,7 & 52,4 & 52,4 \\
\hline Hieracium & Asteraceae & 41 & 0,0 & 51,2 & 70,7 & 7,3 & 92,7 & 61,0 & 51,2 \\
\hline Potentilla & Rosaceae & 41 & 12,2 & 48,8 & 61,0 & 9,8 & 78,0 & 24,4 & 24,4 \\
\hline Trifolium & Fabaceae & 41 & 7,3 & 85,4 & 39,0 & 4,9 & 97,6 & 24,4 & 19,5 \\
\hline
\end{tabular}


pourcentages les plus élevés dans les milieux buissonneux et forestiers, avec respectivement $70,7 \%$ et $55,2 \%$ de taxons présents. Dans ces milieux, les Brassicaceae et les Caryophyllaceae sont les deux seules familles du tableau qui montrent des pourcentages inférieurs à $20 \%$.

\section{Genres}

Parmi les quinze genres réunissant chacun au moins 40 taxons dans les Alpes (AESCHIMANN \& al., 2011a: 39), les Carex montrent aussi bien les proportions les plus élevées de taxons présents dans les milieux marécageux que dans les aquatiques (tableau 7), soit respectivement $54,8 \%$ et $30,4 \%$. Dans les milieux aquatiques, le genre Ranunculus égale ce dernier pourcentage. Dans les milieux marécageux, on note aussi une proportion de présence relativement élevée parmi les taxons du genre Gentiana, soit $36,4 \%$. Ce sont les Trifolium qui affichent les plus hauts pourcentages dans les milieux herbacés et les milieux nitrophiles, avec respectivement $97,6 \%$ et $85,4 \%$. Dans les milieux rocheux, ce sont les Saxifraga, avec 98,3\%, le maximum absolu du tableau. En effet, ce genre compte 47,5\% d'endémiques (AEsCHIMANN $\&$ al., 2011a: 47). Très sténoèces, les Saxifraga comptent moins de $50 \%$ de taxons présents dans chacune des six autres catégories principales de milieux. Dans les milieux rocheux, les Carex ont le plus faible pourcentage, soit 22,6\%. Le genre Centaurea montre la proportion la plus élevée de taxons présents dans les milieux buissonneux, soit $69,8 \%$, alors que cette place revient au genre Viola dans les milieux forestiers, avec 52,4\%. Dans chacun de ces deux milieux, ces genres sont suivis par les Hieracium, avec respectivement $61 \%$ et $51,2 \%$.

\section{Formes biologiques}

Le tableau 8 exprime les pourcentages de taxons présents dans les sept catégories principales de milieux, pour les sept catégories de formes biologiques étudiées précédemment (AEschimAnn \& al., 2012a: 6, 9-11, 19). Les chaméphytes montrent la proportion la plus élevée de taxons présents dans les milieux rocheux, soit $81,3 \%$, ainsi que le profil le plus congruent avec celui des endémiques (tableau 1), chez lesquels on recense précisément un pourcentage élevé de chaméphytes (AESCHIMANN \& al., 2012a: 9). L'importance des chaméphytes dans les milieux limitatifs (parois rocheuses notamment) a déjà été discutée (AEschimANn \& al., 2012b: 203, 210, 214). Les hémicryptophytes montrent un profil assez équilibré, avec la plus forte des proportions de taxons présents dans les milieux herbacés, soit 76,7\%. Les géophytes comptent aussi beaucoup de taxons dans ces milieux $(70,7 \%)$, mais de plus fortes proportions dans les milieux aquatiques, marécageux, buissonneux et forestiers, et en revanche de plus faibles proportions dans les milieux rocheux. Les thérophytes dominent nettement les milieux nitrophiles, avec $88,5 \%$ de taxons présents, surtout dans les cultures, les friches et les milieux rudéraux, mais comptent cependant $47,8 \%$ de taxons dans les milieux herbacés. Les profils des phanérophytes, des hélophytes et des hydrophytes sont conformes aux définitions mêmes de ces formes biologiques. Ainsi, les phanérophytes dominent les milieux buissonneux et forestiers, avec respectivement $79,9 \%$ et $70,7 \%$. Les proportions d'hélophytes sont élevées dans les milieux aquatiques et marécageux, respectivement $93,2 \%$ et $50 \%$. Enfin, les hydrophytes dominent évidemment les milieux aquatiques, avec ici $100 \%$ de taxons présents, le maximum absolu du tableau.

\section{Phytosociologie}

\section{Généralités}

Par niveau syntaxonomique, le tableau 9 regroupe les nombres et pourcentages de taxons ayant un optimum sociologique attribué dans le «Flora alpina». On constate que parmi les taxons indigènes non endémiques, les proportions sont équilibrées entre les deux niveaux supérieurs (classes + ordres $=$ $53 \%$ ) et les deux niveaux inférieurs (alliances + sous-alliances $=47 \%$ ). En revanche, $80 \%$ des endémiques ont un optimum au niveau de l'alliance (53\%) ou de la sous-alliance (27\%).

Tableau 8. - Pourcentages de taxons présents dans les sept catégories principales de milieux, calculés sur les nombres totaux de taxons indiqués entre crochets pour les sept catégories de formes biologiques, ainsi que pour la flore totale. En gras le plus haut pourcentage de chaque colonne, le maximum absolu étant de plus encadré.

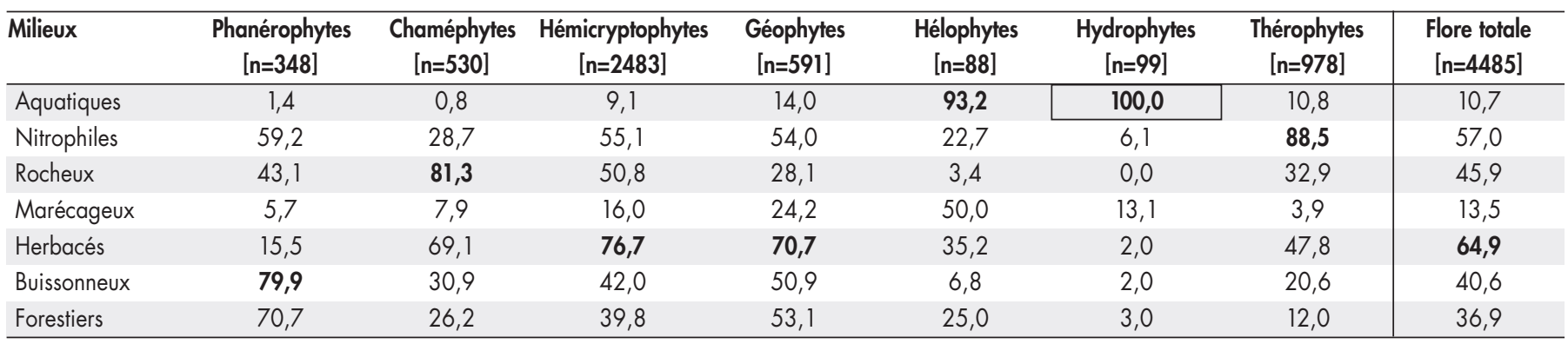


Tableau 9. - Nombres et pourcentages de taxons ayant un optimum sociologique attribué dans le «Flora alpina», par niveau phytosociologique (entre parenthèses le nombre de syntaxons concernés). Pourcentages calculés sur les nombres totaux de taxons indiqués entre crochets pour la flore totale et ses sous-ensembles et arrondis à l'unité.

\begin{tabular}{|c|c|c|c|c|c|c|c|c|c|c|}
\hline \multirow[t]{2}{*}{ Syntaxons } & \multicolumn{2}{|c|}{ Flore totale $[n=4485]$} & \multicolumn{2}{|c|}{ Endémiques [n=501] } & \multicolumn{2}{|c|}{ Indigènes [n=3482] } & \multicolumn{2}{|c|}{$\begin{array}{l}\text { Endémiques+indigènes } \\
\qquad[\mathrm{n}=3983]\end{array}$} & \multicolumn{2}{|c|}{ Xénophytes [n=502. } \\
\hline & $\mathrm{Nb}$. & $\%$ & $\mathrm{Nb}$. & $\%$ & $\mathrm{Nb}$. & $\%$ & $\mathrm{Nb}$. & $\%$ & $\mathrm{Nb}$. & $\%$ \\
\hline Classes (50) & 1250 & 28 & 47 & 9 & 1050 & 30 & 1097 & 27 & 153 & 31 \\
\hline Alliances (182) & 1971 & 44 & 267 & 53 & 1526 & 44 & 1793 & 45 & 178 & 35 \\
\hline Sous-alliances (52) & 241 & 5 & 132 & 27 & 108 & 3 & 240 & 6 & 1 & 0 \\
\hline
\end{tabular}

Tableau 10. - Pourcentages de taxons ayant leur optimum sociologique dans chaque classe ou les syntaxons subordonnés, calculés sur les nombres totaux de taxons indiqués entre crochets pour la flore totale (classement décroissant) et ses sous-ensembles. En gras les pourcentages $>20 \%$, le maximum absolu étant de plus encadré. Les 25 classes dont le pourcentage est $\geq 1 \%$ pour la flore totale ou les endémiques déterminent le nombre de lignes.

\begin{tabular}{|c|c|c|c|c|c|c|}
\hline № Synsystème & Classe & $\begin{array}{c}\text { Flore totale } \\
{[n=4485]}\end{array}$ & $\begin{array}{c}\text { Endémiques } \\
{[n=501]}\end{array}$ & $\begin{array}{l}\text { Indigènes } \\
{[n=3482]}\end{array}$ & $\begin{array}{c}\text { Endémiques+ } \\
\text { indigènes } \\
\text { [n=3983] }\end{array}$ & $\begin{array}{c}\text { Xénophyłes } \\
{[n=502]}\end{array}$ \\
\hline 9.1 & Festuco-Brometea & 11,8 & 11,0 & 13,3 & 13,0 & 2,0 \\
\hline 11.2 & Molinio-Arrhenatheretea & 7,0 & 0,8 & 8,4 & 7,4 & 3,4 \\
\hline 3.2 & Asplenietea trichomanis & 6,1 & 22,6 & 4,0 & 6,4 & 3,6 \\
\hline 3.3 & Thlaspietea rotundifolii & 5,9 & 20,6 & 4,5 & 6,5 & 0,8 \\
\hline 5.1 & Artemisietea vulgaris & 4,7 & 1,0 & 4,2 & 3,8 & 12,5 \\
\hline 11.1 & Trifolio-Geranietea & 3,6 & 1,6 & 4,4 & 4,0 & 0,2 \\
\hline 10.4 & Juncetea trifidi & 3,5 & 8,8 & 3,2 & 3,9 & 0,0 \\
\hline 4.1 & Koelerio-Corynephoretea & 2,9 & 1,2 & 3,4 & 3,1 & 0,8 \\
\hline 11.5 & Mulgedio-Aconitetea & 2,1 & 3,8 & 2,1 & 2,3 & 0,4 \\
\hline 13.3 & Crataego-Prunetea & 2,0 & 0,8 & 2,0 & 1,8 & 3,8 \\
\hline 14.8 & Quercetea pubescentis & 1,9 & 0,2 & 2,3 & 2,0 & 1,0 \\
\hline 11.3 & Filipendulo-Convolvuletea & 1,4 & 0,0 & 1,2 & 1,1 & 4,0 \\
\hline 1.3 & Potametea pectinati & 1,3 & 0,0 & 1,5 & 1,3 & 1,2 \\
\hline 14.3 & Vaccinio-Piceetea excelsae & 1,2 & 1,0 & 1,4 & 1,4 & 0,0 \\
\hline 9.2 & Lygeo-Stipetea & 1,1 & 0,0 & 1,3 & 1,1 & 1,0 \\
\hline 2.2 & Isoëto-Nanojuncetea & 1,0 & 0,0 & 1,3 & 1,2 & 0,2 \\
\hline 12.4 & Rosmarinetea & 1,0 & 0,4 & 1,2 & 1,1 & 0,2 \\
\hline 14.2 & Erico-Pinetea & 0,8 & 1,4 & 0,8 & 0,9 & 0,0 \\
\hline 13.4 & Betulo carpaticae-Alnetea viridis & 0,5 & 1,0 & 0,5 & 0,6 & 0,0 \\
\hline
\end{tabular}


La sténoécité prononcée des endémiques, mise en évidence à propos des milieux, est ainsi corroborée. A l'inverse, seulement $35 \%$ des xénophytes ont un optimum dans les deux niveaux inférieurs et $48 \%$ trouvent leur optimum dans les deux niveaux supérieurs. Cependant, dans toutes les catégories, le niveau de l'alliance est celui qui rassemble le plus de taxons. A l'exception des xénophytes, ceci est encore davantage le cas si l'on regroupe les deux niveaux de l'alliance et de la sousalliance. Ainsi, la moitié des taxons de la flore totale et de la flore indigène totale ont leur optimum sociologique au niveau de l'alliance. La totalité des taxons sans optimum sociologique attribué dans les Alpes sont des xénophytes cultivés (utilitaires et ornementaux).

\section{Classes}

La moitié des cinquante classes du synsystème figure sur le tableau 10, où chaque résultat est la somme des pourcentages de taxons ayant leur optimum dans la classe elle-même ou dans les syntaxons subordonnés. Parmi les 3482 taxons indigènes non endémiques recensés dans le «Flora alpina», $38,9 \%$ ont leur optimum sociologique dans les huit classes de végétation herbacée présentes sur le tableau (appartenant aux formations 9, 10 et 11 du synsystème), dont les FestucoBrometea (13,3\%), les Molinio-Arrhenatheretea ( $8,4 \%)$, les Elyno-Seslerietea variae (5\%) et les Trifolio-Geranietea $(4,4 \%)$, ce qui confirme l'importance des milieux herbacés mise en évidence dans le tableau 1. Deux classes des mauvaises herbes des cultures et de végétation rudérale rassemblent 13,7\% des taxons, respectivement les Stellarietea mediae (9,5\%) et les Artemisietea vulgaris (4,2\%), en accord avec le second rang occupé par les milieux nitrophiles.

Parmi les taxons endémiques, la préférence pour les milieux rocheux et les éboulis (tableaux 1 et 2) se retrouve au niveau du tableau 10, puisque trois classes rassemblent 44,4\% des taxons, les Asplenietea trichomanis (22,6\%), les Thlaspietea rotundifolii $(20,6 \%)$ et les Koelerio-Corynephoretea $(1,2 \%)$. Ces classes correspondent respectivement aux communautés végétales des fissures de rochers (chasmophytes), des éboulis et des affleurements rocheux. Les pourcentages sont en accord avec les estimations de PAWLOWSKI (1970: 213), qui admet que le quart ou le cinquième des endémiques de l'arc alpin sont des espèces des fentes de rochers et que cette proportion augmente probablement à 35 $40 \%$ lorsqu'on ajoute les plantes des éboulis. Cela confirme également l'affirmation de BRAUN-BLANQUET (1948: 26) selon laquelle «La végétation rupicole de la Classe des Asplenietea du pourtour méditerranéen, fortement différenciée, se distingue par sa grande richesse en espèces endémiques». Cependant, on relève aussi la proportion très élevée $(44,6 \%)$ d'endémiques ayant leur optimum dans les milieux herbacés (formations 9, 10 et 11 du synsystème), avec notamment les quatre classes Elyno-Seslerietea variae (pelouses subalpinesalpines calcaires, 18,6\%), Festuco-Brometea (prés secs, $11 \%$ ), Juncetea trifidi (pelouses subalpines-alpines siliceuses, $8,8 \%$ ) et Mulgedio-Aconitetea (mégaphorbiées, 3,8\%). Au total, neuf classes des milieux rocheux et des milieux herbacés regroupent $89 \%$ des endémiques.

La forte tendance nitrophile des xénophytes (tableaux 1 et 2) est vérifiée, puisque deux classes rassemblent $44,6 \%$ des taxons, les Stellarietea mediae (32,1\%, maximum absolu du tableau 10) et les Artemisietea vulgaris (12,5\%).

\section{Alliances et sous-alliances}

Les principales alliances figurent dans la partie supérieure du tableau 11, où chaque résultat est la somme des pourcentages de taxons ayant leur optimum sociologique dans l'alliance elle-même ou dans les sous-alliances subordonnées. La sténoécité des endémiques est ici encore avérée, puisque plus du tiers des endémiques (34\%) ont leur optimum dans les trois premières alliances du tableau 11 et plus de la moitié $(50,8 \%)$ dans les neuf premières (en gras). La sténoécité est remarquable pour les milieux rocheux et les éboulis, puisque les premiers comprennent $20 \%$ des taxons ayant leur optimum dans les deux alliances Potentillion caulescentis (calcaire; 16,2\%) et Androsacion vandellii (silice; 3,8\%), et les deuxièmes $15,8 \%$ des taxons dans les trois alliances Thlaspion rotundifolii (calcaire; 6,2\%), Drabion hoppeanae (calcschistes; $3 \%$ ) et Androsacion alpinae (silice; $3 \%$ ). Ces pourcentages sont à peine plus bas qu'au niveau de la classe (milieux rocheux: $22,6 \%$, éboulis: $20,6 \%$; tableau 10 ). La sténoécité est également élevée pour les pelouses subalpines-alpines. En particulier, $11,6 \%$ des endémiques trouvent leur optimum dans les pelouses calcaires du Seslerion variae (thermophiles) et 2,6\% dans celles du Caricion ferrugineae (mésophiles). De plus, $2,4 \%$ des endémiques trouvent leur optimum dans les pelouses sur silice du Festucion variae (thermophiles) et 1,6\% dans celles du Caricion curvulae (mésophiles).

Les principales sous-alliances figurent dans la partie inférieure du tableau 11. Les milieux rocheux regroupent encore $16,2 \%$ des endémiques, dont $13,6 \%$ ayant leur optimum sociologique sur calcaire (Physoplexido-Potentillenion, Saxifragenion lingulatae et Potentillenion caulescentis) et 2,6\% sur silice (Androsacenion vandellii et Saxifragenion pedemontanae). Plus d'un taxon endémique sur cinq (21\%) trouve son optimum dans les huit premières sous-alliances de la partie inférieure du tableau 11 (en gras). Parmi les 31 endémiques $(6,2 \%)$ ayant leur optimum dans le Physoplexido-Potentillenion, 13 ne se rencontrent en principe pas ailleurs que dans cette sous-alliance et sont donc étroitement sténoèces (sousalliance mentionnée sans parenthèses dans le «Flora alpina»; voir ci-après la liste de ces 13 taxons, sous «4. Patrons cartographiques»). De même, parmi les 20 endémiques (4\%) ayant 
Tableau 11. - Pourcentages de taxons ayant leur optimum sociologique dans chaque alliance ou les sous-alliances subordonnées (partie supérieure), ainsi que dans chaque sous-alliance (partie inférieure), calculés sur les nombres totaux de taxons indiqués entre crochets pour la flore totale et ses sous-ensembles (classements décroissants selon les pourcentages d'endémiques). Le maximum absolu de chaque partie est encadré. Partie supérieure: en gras les pourcentages $\geq 2 \%$; les 22 alliances dont le pourcentage est $\geq 1 \%$ pour la flore totale ou les endémiques ou $>2 \%$ pour les xénophytes déterminent le nombre de lignes. Partie inférieure: en gras les pourcentages $\geq 1 \%$; les 11 sous-alliances dont le pourcentage est $>0,5 \%$ pour les endémiques déterminent le nombre de lignes.

\begin{tabular}{|c|c|c|c|c|c|c|}
\hline № Synsystème & Alliance & $\begin{array}{c}\text { Flore totale } \\
{[n=4485]}\end{array}$ & $\begin{array}{c}\text { Endémiques } \\
{[n=501]}\end{array}$ & $\begin{array}{l}\text { Indigènes } \\
{[n=3482]}\end{array}$ & $\begin{array}{c}\text { Endémiques+ } \\
\text { indigènes } \\
{[n=3983]}\end{array}$ & $\begin{array}{c}\text { Xénophytes } \\
{[n=502]}\end{array}$ \\
\hline 3.211 & Potentillion caulescentis & 2,8 & 16,2 & 1,3 & 3,1 & 0,0 \\
\hline 10.211 & Seslerion variae & 2,8 & 11,6 & 2,0 & 3,2 & 0,0 \\
\hline 3.311 & Thlaspion rotundifolii & 1,0 & 6,2 & 0,4 & 1,2 & 0,0 \\
\hline 3.221 & Androsacion vandellii & 0,9 & 3,8 & 0,6 & 1,0 & 0,0 \\
\hline 3.331 & Drabion hoppeanae & 0,4 & 3,0 & 0,1 & 0,5 & 0,0 \\
\hline 3.341 & Androsacion alpinae & 0,7 & 3,0 & 0,5 & 0,8 & 0,0 \\
\hline 10.212 & Caricion ferrugineae & 0,8 & 2,6 & 0,6 & 0,9 & 0,2 \\
\hline 10.421 & Festucion variae & 0,5 & 2,4 & 0,3 & 0,6 & 0,0 \\
\hline 11.514 & Adenostylion & 0,8 & 2,0 & 0,7 & 0,9 & 0,4 \\
\hline 3.312 & Petasition paradoxi & 0,8 & 1,8 & 0,8 & 0,9 & 0,0 \\
\hline 3.321 & Arabidion caeruleae & 0,4 & 1,8 & 0,3 & 0,5 & 0,0 \\
\hline 9.113 & Stipo-Poion xerophilae & 0,4 & 1,6 & 0,3 & 0,5 & 0,4 \\
\hline 10.411 & Caricion curvulae & 0,5 & 1,6 & 0,5 & 0,6 & 0,0 \\
\hline 9.142 & Avenion sempervirentis & 0,2 & 1,4 & 0,1 & 0,3 & 0,0 \\
\hline 11.112 & Trifolion medii & 0,5 & 1,0 & 0,5 & 0,6 & 0,0 \\
\hline 14.211 & Erico-Pinion & 0,3 & 1,0 & 0,3 & 0,4 & 0,0 \\
\hline 13.312 & Berberidion vulgaris & 1,3 & 0,8 & 1,2 & 1,2 & 2,4 \\
\hline 2.312 & Caucalidion lappulae & 1,2 & 0,4 & 1,3 & 1,2 & 0,8 \\
\hline 2.343 & Sisymbrion & 1,0 & 0,0 & 0,7 & 0,7 & 3,6 \\
\hline 2.351 & Polygonion avicularis & 0,4 & 0,0 & 0,2 & 0,2 & 2,2 \\
\hline 11.111 & Geranion sanguinei & 2,1 & 0,0 & 2,6 & 2,3 & 0,2 \\
\hline \multirow[t]{2}{*}{11.311} & Convolvulion sepium & 0,6 & 0,0 & 0,3 & 0,3 & 3,0 \\
\hline & Sous-alliance & & & & & \\
\hline 3.211 .3 & Physoplexido-Potentillenion & 0,8 & 6,2 & 0,2 & 0,9 & 0,0 \\
\hline 3.211 .4 & Saxifragenion lingulatae & 0,5 & 4,0 & 0,1 & 0,6 & 0,0 \\
\hline 3.211 .1 & Potentillenion caulescentis & 0,5 & 3,4 & 0,2 & 0,6 & 0,0 \\
\hline 10.211 .6 & Caricenion austroalpinae & 0,4 & 2,6 & 0,2 & 0,5 & 0,0 \\
\hline 3.221 .1 & Androsacenion vandellii & 0,3 & 1,4 & 0,2 & 0,3 & 0,0 \\
\hline 3.221 .2 & Saxifragenion pedemontanae & 0,2 & 1,2 & 0,0 & 0,2 & 0,0 \\
\hline 10.211 .4 & Caricenion firmae & 0,2 & 1,2 & 0,1 & 0,3 & 0,0 \\
\hline 10.211 .3 & Drabo-Seslerienion variae & 0,2 & 1,0 & 0,1 & 0,2 & 0,0 \\
\hline 9.113 .2 & Stipo-Poenion perconcinnae & 0,2 & 0,8 & 0,1 & 0,2 & 0,0 \\
\hline 10.211.1 & Seslerienion variae & 0,2 & 0,8 & 0,1 & 0,2 & 0,0 \\
\hline 9.122 .1 & Hypochoeridenion maculatae & 0,1 & 0,6 & 0,1 & 0,1 & 0,0 \\
\hline
\end{tabular}


leur optimum dans le Saxifragenion lingulatae, huit sont étroitement sténoèces. Il n'y a en revanche qu'un taxon étroitement sténoèce parmi les six endémiques $(1,2 \%)$ de la sous-alliance Saxifragenion pedemontanae, à savoir Saxifraga florulenta, et aucun parmi les treize endémiques $(2,6 \%)$ de la sous-alliance Caricenion austroalpinae. La présence de plusieurs taxons endémiques dans les sous-alliances évoquées, dont certains étroitement sténoèces, justifie pleinement leur individualisation d'un point de vue phytosociologique. Ces quatre sousalliances sont endémiques des Alpes.

D'autre part, il est important de noter que $13,7 \%$ des 241 taxons (tableau 9) ayant un optimum au niveau de la sousalliance sont étroitement sténoèces. Parmi ces 33 taxons à fidélité absolue à une et une seule sous-alliance, 26 sont des endémiques, soit quatre sur cinq. Autrement dit, parmi les 132 endémiques ayant un optimum au niveau de la sousalliance, un sur cinq est étroitement sténoèce. Seules deux sous-alliances rassemblent chacune plus d'un endémique étroitement sténoèce, le Physoplexido-Potentillenion et le Saxifragenion lingulatae.

\section{Patrons cartographiques}

Les patrons cartographiques des quatre sous-alliances mentionnées plus haut sont particulièrement éloquents (fig. 2-5) et documentent les deux principaux foyers d'endémisme précédemment évoqués (AESCHIMANN \& al., 2011a: 49-53).

La distribution du Physoplexido-Potentillenion (fig. 2), qui occupe les rochers calcaires, souvent dolomitiques, de la partie sud des Alpes orientales (SUTTER, 1969; THEURILLAT \& al., $1995)$ correspond exactement au vaste regroupement des divisions administratives 17, 20, 21, 22, 23, 25 et 26 (AESCHIMANN $\&$ al., 2011a: 50; 2012b: 208). Ce patron souligne le «compartiment floristique» situé au sud de la faille périadriatique (ligne Adda - Drave), où dominent les roches sédimentaires, du lac de Côme à la Slovénie. Cela correspond en large partie à la «province insubrico-carnique» de VIERHAPPER (1924, 1925). Trente-sept taxons ont leur optimum dans le Physoplexido-Potentillenion, dont 14 sont étroitement sténoèces (38\%). Voici les 31 endémiques ayant leur optimum dans cette sous-alliance, dont 13 sont étroitement sténoèces (marqués d'un astérisque): Aquilegia thalictrifolia, *Arenaria huteri, *Asplenium presolanense, Campanula cespitosa, C. elatinoides, C. petraea, C. raineri, *Daphne petraea, Erysimum aurantiacum, Medicago pironae, Minuartia graminifolia, M. grignensis, *Moehringia bavarica subsp. insubrica, ${ }^{*}$ M. dielsiana, ${ }^{*}$. glaucovirens, ${ }^{*}$ M. villosa, ${ }^{*}$ Physoplexis comosa, *Primula albenensis, *P. grignensis, P. recubariensis, P. spectabilis, P. tyrolensis, *Saxifraga arachnoidea, S. tenella, *S. tombeanensis, *S. vandellii, Silene elisabethae, S. veselskyi, Spiraea decumbens subsp. decumbens, S. decumbens subsp. hacquetii et Telekia speciosissima.
Le patron du Caricenion austroalpinae (fig. 3 ) est très comparable au précédent. En effet, cette sous-alliance occupe les pelouses calcaires subalpines (SUTTER, 1962, 1969; FEOLI Chiapella \& Poldini, 1994) de la bordure sud des Alpes orientales. Dix-neuf taxons y trouvent leur optimum, dont les 13 endémiques suivants: Allium insubricum, Biscutella prealpina, Callianthemum kernerianum, Carex ferruginea subsp. austroalpina, Centaurea rhaetica, Euphorbia variabilis, Festuca alpestris, F. calva, Helictotrichon petzense, Hypochaeris facchiniana, Primula glaucescens, Viola culminis et V. dubyana.

A l'extrémité sud-occidentale des Alpes, le patron du Saxifragenion lingulatae (fig. 4), sous-alliance des rochers calcaires collinéens-subalpins (RIOUX \& QuÉzeL, 1949; QuÉZEL, 1951; BARBERO, 1966; THEURILLAT \& al., 1995), correspond exactement au regroupement des divisions 1, 2 et 3 (AEsCHIMANN \& al., 2011a: 50). Ce patron souligne le foyer d'endémisme des Alpes-Maritimes et régions voisines (OzENDA, 1951; BARBERO, 1967; MarTini, 1984, 1997). Vingt-deux taxons ont leur optimum dans le Saxifragenion lingulatae, dont les 20 endémiques suivants, parmi lesquels huit sont étroitement sténoèces (marqués d'un astérisque): Alyssum ligusticum, Asperula hexaphylla, *Asplenium jahandiezii, Ballota frutescens, Campanula albicans, C. fritschii, C. longisepala, C. macrorhiza, Helianthemum lunulatum, Micromeria marginata, *Moehringia intermedia, *M. lebrunii, *M. sedoides, *Phyteuma cordatum, *Ph. villarsii, *Potentilla saxifraga, *Primula allionii, Saxifraga cochlearis, Sedum fragrans et Silene campanula. A noter que Saxifraga callosa (= lingulata) n'est ni étroitement sténoèce de la sous-alliance, ni endémique de l'arc alpin, puisque son aire de distribution s'étend à l'Apennin.

La sous-alliance du Saxifragenion pedemontanae (fig. 5) occupe les rochers siliceux subalpins-alpins à l'extrémité sudoccidentale des Alpes (Theurillat \& al., 1995). Son patron cartographique est comparable au précédent, mais son centre de gravité se situe sur le versant italien (Piémont), où dominent les substrats siliceux (AESCHIMANN \& al., 2012b: 205208). Sept taxons ont leur optimum dans le Saxifragenion pedemontanae, dont les six endémiques suivants, un seul étant étroitement sténoèce (marqué d'un astérisque): Galium tendae, Jovibarba allionii, *Saxifraga florulenta, Saxifraga pedemontana, Senecio persoonii et Silene cordifolia.

\section{Etages de végétation}

En fonction de l'altitude, le tableau 12 exprime les proportions de taxons ayant leur optimum sociologique au sein des principales classes, qui peuvent être assemblées en trois groupes. Le premier comprend seize classes dont le centre de gravité se situe aux étages collinéen et montagnard, et pour lesquelles le pourcentage diminue jusqu'à l'étage nival où il est le plus souvent nul. Ce sont les classes des Thero-Brachypodietea 


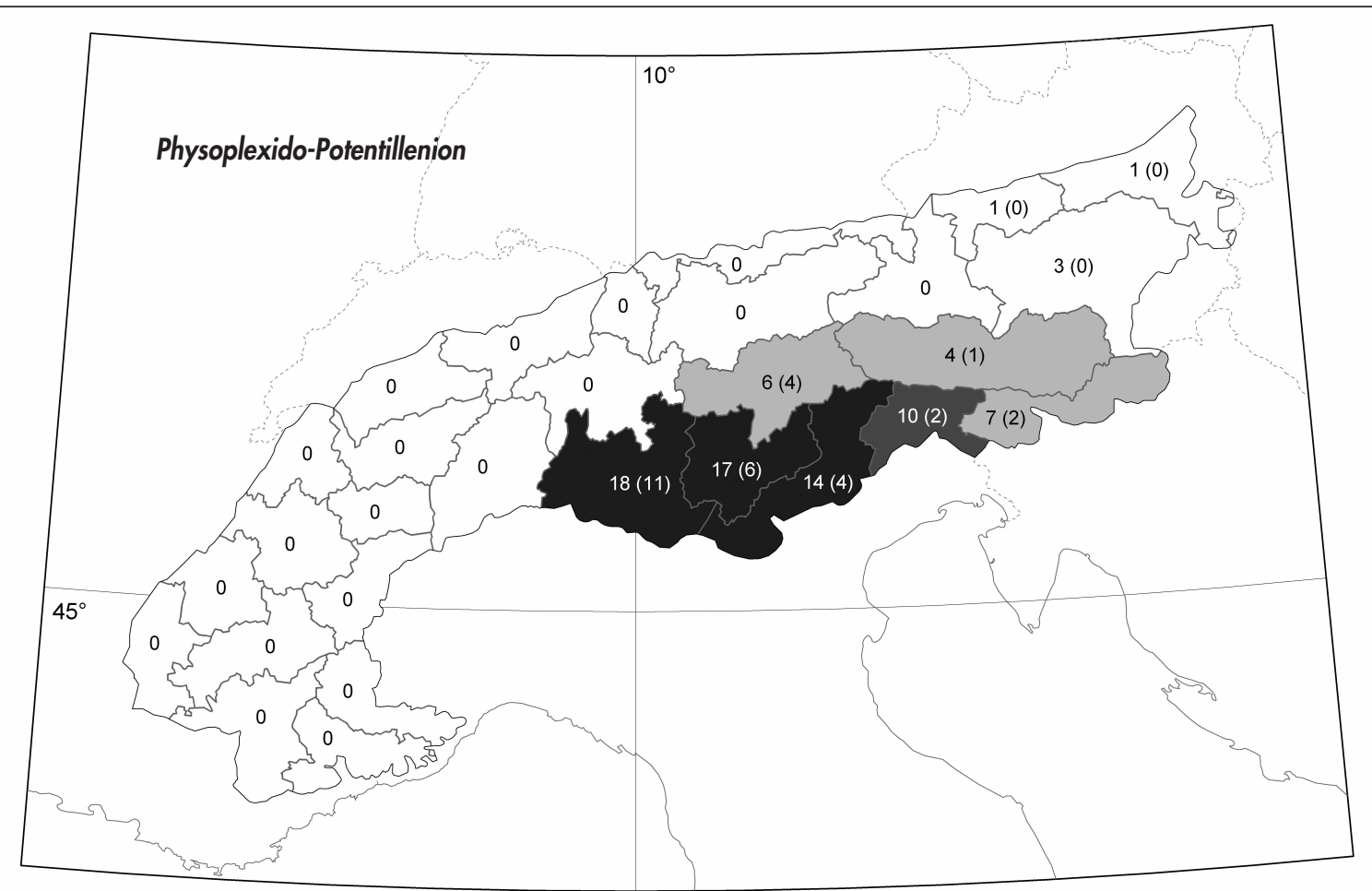

Fig. 2. - Carte de la dition avec, par division administrative, le nombre de taxons endémiques de l'arc alpin ayant leur optimum sociologique dans la sous-alliance du PhysoplexidoPotentillenion ( $n \circ$ 3.211.3). Entre parenthèses le nombre d'endémiques étroitement sténoèces (fidélité absolue à la sous-alliance). Quatre classes sont définies selon les nombres de taxons présents: blanc: $0-3$; gris clair: $4-7$; gris foncé: $8-11$; noir: $\geq 12$

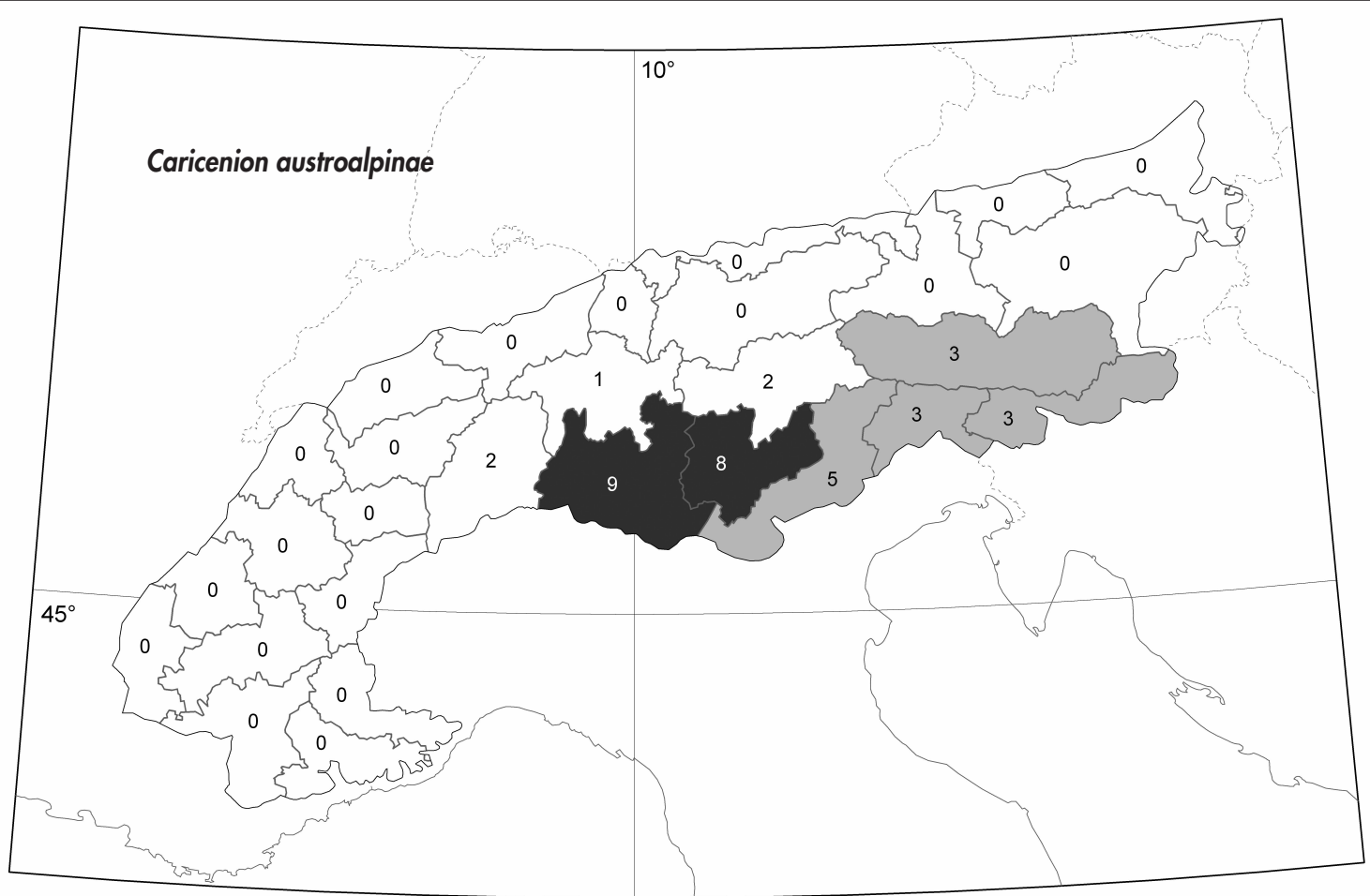

Fig. 3. - Carte de la dition avec, par division administrative, le nombre de taxons endémiques de l'arc alpin ayant leur optimum sociologique dans la sous-alliance du Caricenion austroalpinae (n॰ 10.211.6). Trois classes sont définies selon les nombres de taxons présents: blanc: 0-2; gris clair: $3-5$; gris foncé: $\geq 6$. 


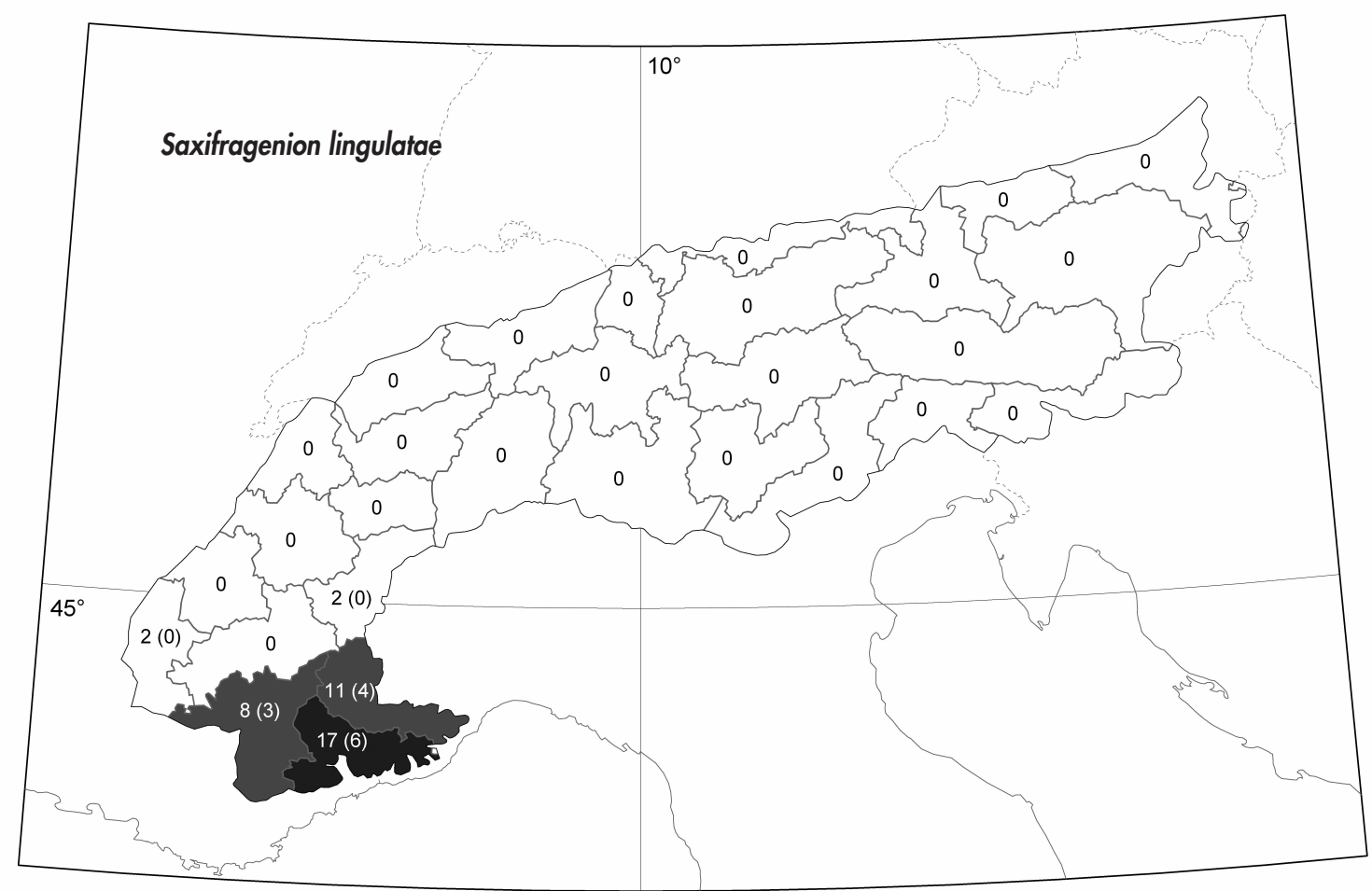

Fig. 4. - Carte de la dition avec, par division administrative, le nombre de taxons endémiques de l'arc alpin ayant leur optimum sociologique dans la sous-alliance du Saxifragenion lingulatae ( $\mathrm{n} \circ 3.211 .4)$. Entre parenthèses le nombre d'endémiques étroitement sténoèces (fidélité absolue à la sous-alliance). Quatre classes sont définies selon les nombres de taxons présents: blanc: $0-3$; gris clair: $4-7$; gris foncé: $8-11$; noir: $\geq 12$.

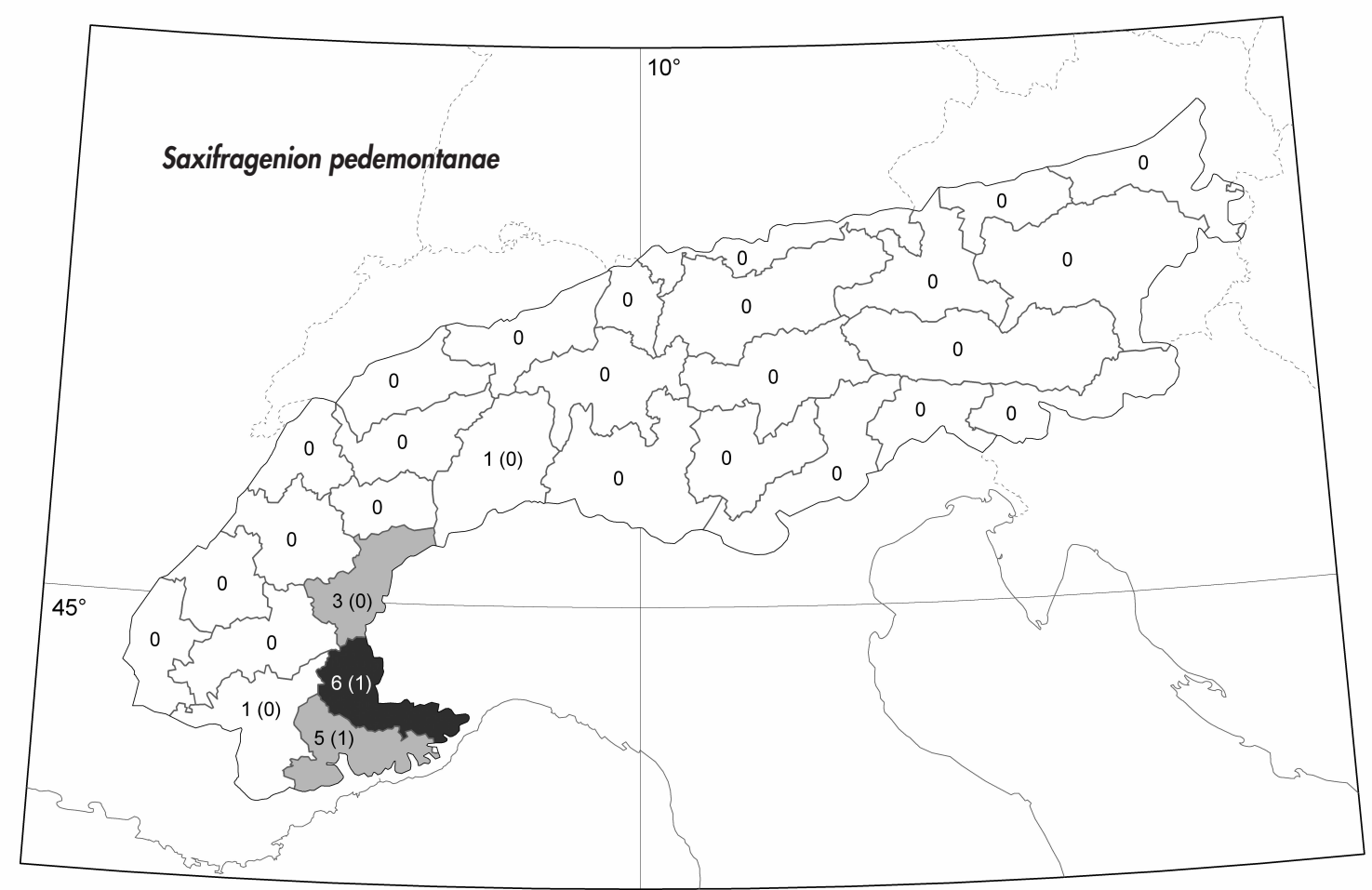

Fig. 5. - Carte de la dition avec, par division administrative, le nombre de taxons endémiques de l'arc alpin ayant leur optimum sociologique dans la sous-alliance du Saxifragenion pedemontanae ( $n^{\circ} 3.221 .2$ ). Entre parenthèses le nombre d'endémiques étroitement sténoèces (fidélité absolue à la sous-alliance). Trois classes sont définies selon les nombres de taxons présents: blanc: $0-2$; gris clair: $3-5$; gris foncé: $\geq 6$. 
Tableau 12. - Pourcentages de taxons ayant potentiellement leur optimum sociologique dans chaque classe ou ses syntaxons subordonnés, calculés sur les nombres totaux de taxons indiqués entre crochets pour chaque étage de végétation, ainsi que pour la flore totale. Voir le texte pour la signification des trois groupes. Première clé de tri : groupes; seconde clé de tri : classement décroissant selon les valeurs dans les encadrés. En gras les pourcentages $>10 \%$, le maximum absolu étant de plus encadré en gras. Le choix des classes est identique à celui du tableau 10, mais deux classes dont le pourcentage $>3 \%$ à l'étage nival sont ajoutées, les Carici rupestrisKobresietea bellardii et les Salicetea herbaceae.

\begin{tabular}{|c|c|c|c|c|c|c|c|c|}
\hline № Synsystème & Classe & Groupe & $\begin{array}{l}\text { Collinéen } \\
{[n=3515]}\end{array}$ & $\begin{array}{c}\text { Montagnard } \\
{[n=3325]}\end{array}$ & $\begin{array}{l}\text { Subalpin } \\
{[n=2400]}\end{array}$ & $\begin{array}{c}\text { Alpin } \\
{[n=1099]}\end{array}$ & $\begin{array}{c}\text { Nival } \\
{[n=152]}\end{array}$ & $\begin{array}{c}\text { Flore totale } \\
{[n=4485]}\end{array}$ \\
\hline 2.3 & Stellarietea mediae & 1 & 14,0 & 9,5 & 5,1 & 1,1 & 0,0 & 11,0 \\
\hline 9.1 & Festuco-Brometea & 1 & 13,8 & 13,9 & 11,2 & 4,7 & 0,7 & 11,8 \\
\hline 11.2 & Molinio-Arrhenatheretea & 1 & 8,0 & 7,8 & 7,5 & 6,0 & 1,3 & 7,0 \\
\hline 14.7 & Carpino-Fagetea sylvaticae & 1 & 7,3 & 7,4 & 6,0 & 1,4 & 0,0 & 6,0 \\
\hline 5.1 & Artemisietea vulgaris & 1 & 5,8 & 4,9 & 3,6 & 1,0 & 0,0 & 4,7 \\
\hline 4.2 & Thero-Brachypodietea & 1 & 2,9 & 1,3 & 0,1 & 0,0 & 0,0 & 2,3 \\
\hline 6.1 & Phragmito-Magnocaricetea & 1 & 2,6 & 2,0 & 1,0 & 0,2 & 0,0 & 2,1 \\
\hline 14.8 & Quercetea pubescentis & 1 & 2,4 & 2,1 & 0,8 & 0,0 & 0,0 & 1,9 \\
\hline 13.3 & Crataego-Prunetea & 1 & 2,3 & 2,4 & 1,7 & 0,5 & 0,0 & 2,0 \\
\hline 11.3 & Filipendulo-Convolvuletea & 1 & 1,8 & 1,3 & 0,8 & 0,1 & 0,0 & 1,4 \\
\hline 12.4 & Rosmarinetea & 1 & 1,3 & 0,8 & 0,5 & 0,1 & 0,0 & 1,0 \\
\hline 3.3 & Thlaspietea rotundifolii & 2 & 2,3 & 4,5 & 9,3 & 16,8 & 40,1 & 5,9 \\
\hline 10.4 & Juncetea trifidi & 2 & 0,6 & 2,6 & 6,2 & 12,8 & 21,7 & 3,5 \\
\hline 3.2 & Asplenietea trichomanis & 2 & 5,0 & 6,1 & 8,0 & 10,7 & 14,5 & 6,1 \\
\hline 10.3 & Carici rupestris-Kobresietea bellardii & 2 & 0,1 & 0,2 & 1,0 & 2,3 & 5,9 & 0,6 \\
\hline 8.1 & Salicetea herbaceae & 2 & 0,0 & 0,2 & 0,6 & 1,5 & 3,9 & 0,4 \\
\hline 14.2 & Erico-Pinetea & 3 & 0,8 & 1,0 & 0,8 & 0,5 & 0,0 & 0,8 \\
\hline 11.5 & Mulgedio-Aconitetea & 3 & 0,9 & 2,6 & 3,8 & 3,1 & 0,0 & 2,1 \\
\hline 14.3 & Vaccinio-Piceetea excelsae & 3 & 1,1 & 1,5 & 2,2 & 1,8 & 0,7 & 1,2 \\
\hline 10.2 & Elyno-Seslerietea variae & 3 & 1,7 & 5,6 & 11,1 & 20,7 & 8,6 & 6,0 \\
\hline 7.2 & Scheuchzerio-Caricetea fuscae & 3 & 2,1 & 2,8 & 3,6 & 4,8 & 0,0 & 2,3 \\
\hline
\end{tabular}

(milieux pionniers à thérophytes et succulentes), des FestucoBrometea et Lygeo-Stipetea (prés secs), des Molinio-Arrhenatheretea (prés et pâturages méso-hygrophiles), des Stellarietea mediae et Artemisietea vulgaris (cultures et végétation rudérale), des Potametea pectinati (milieux aquatiques permanents), des Isoëto-Nanojuncetea (milieux aquatiques temporaires), des Phragmito-Magnocaricetea (milieux semi-aquatiques), des Filipendulo-Convolvuletea (mégaphorbiées nitrophiles humides), des Rosmarinetea (garrigues) et des Quercetea pubescentis (chênaies et ostryaies thermophiles). La présence de quelques taxons de ces classes à l'étage nival s'explique par un optimum sociologique différent à haute altitude de taxons à large distribution altitudinale: Erysimum sylvestre pour la classe des FestucoBrometea, ainsi que Leontodon hispidus et Poa alpina pour la classe des Molinio-Arrhenatheretea. Dans cette optique, la classe des Koelerio-Corynephoretea (affleurements rocheux) peut elle aussi être rangée dans ce premier groupe des classes dont le pourcentage diminue avec l'altitude, car l'augmentation de son pourcentage à l'étage nival est due à la présence des trois sous-espèces stiriacum, burnatii et montanum du Sempervivum montanum. On range également dans le premier groupe trois classes dont le pourcentage le plus élevé est à l'étage montagnard, mais presque égal à celui relevé au collinéen. Il s'agit de végétations forestières et des végétations arbustives et herbacées qui leur sont liées: les Carpino-Fagetea sylvaticae (forêts décidues mésophiles), les Crataego-Prunetea (buissons méso-thermophiles) et les Trifolio-Geranietea (ourlets mésothermophiles). Le second groupe correspond à cinq classes dont 
la proportion de taxons augmente régulièrement avec l'altitude: les Asplenietea trichomanis (fissures de rochers), les Thlaspietea rotundifolii (éboulis) et les Juncetea trifidi (pelouses subalpines-alpines sur silice), ainsi que les Carici rupestris-Kobresietea bellardii (pelouses alpines ventées et peu enneigées) et les Salicetea herbaceae (combes à neige alpines sur sol acide). Le maximum absolu du tableau 12 à $40,1 \%$ atteint à l'étage nival par les Thlaspietea rotundifolii corrobore celui du tableau 4, soit $89,5 \%$ pour les «éboulis s.l.». Les distributions dans les six classes restantes (troisième groupe) présentent un mode à l'étage montagnard pour les Erico-Pinetea (pinèdes méso-thermophiles), un mode à l'étage subalpin pour les Vaccinio-Piceetea excelsae (forêts montagnardes-subalpines de conifères) et les Mulgedio-Aconitetea (mégaphorbiées subalpines), et un mode à l'étage alpin pour les Betulo carpaticae-Alnetea viridis (aulnaies vertes et saulaies subalpines), les Elyno-Seslerietea (pelouses subalpines-alpines sur calcaire) et les Scheuchzerio-Caricetea fuscae (bas-marais). Le taxon des forêts de conifères (VaccinioPiceetea excelsae) atteignant l'étage nival avec un changement d'optimum est Homogyne alpina.

La plupart des tendances altitudinales observées vont dans le même sens que celles pour les milieux correspondants, mais il ne s'agit cependant pas d'une circularité. En effet, la majeure partie des taxons ayant un optimum dans une classe donnée en déborde et se retrouve dans d'autres milieux, dominés par d'autres classes. Un milieu comprend donc bien davantage de taxons que ceux ayant leur optimum dans la classe phytosociologique dominante dans le milieu en question. Ainsi, sur l'ensemble de la flore, 11,8\% des taxons (tableau 10) ont un optimum sociologique dans la classe des Festuco-Brometea, mais 32,9\% (tableau 2) se rencontrent dans les «pelouses, prés et pâturages secs», qui sont l'apanage de cette classe, soit près de trois fois plus. Dans les deux cas, les proportions des taxons diminuent avec l'altitude (tableaux 4 et 12), mais bien que les deux diminutions soient fortement corrélées, elles ne sont cependant pas parallèles.
Aux étages alpin et nival, plus de $60 \%$ des taxons ont leur optimum sociologique dans quatre classes seulement ( $61 \%$ à l'étage alpin et $85 \%$ au nival). Ces classes de végétations de pelouses subalpines-alpines, d'éboulis et de rochers sont les Elyno-Seslerietea variae, les Juncetea trifidi, les Thlaspietea rotundifolii et les Asplenietea trichomanis. En revanche, ces quatre classes ne rassemblent qu'un taxon sur trois à l'étage subalpin. D'autre part, aux étages collinéen et montagnard, en moyenne deux taxons sur trois se répartissent dans le vaste premier groupe de seize classes. La diversité des optimums sociologiques diminue donc nettement en fonction de l'altitude.

Pour les six classes réunissant chacune plus de 3\% des taxons endémiques (tableau 10, au total un peu plus de $85 \%$ des endémiques), les nombres totaux d'endémiques par étage sont listés dans le tableau 13 , avec en regard la flore indigène non endémique pour comparaison ( $32 \%$ de taxons concernés). Ces distributions altitudinales des optimums sociologiques montrent une concentration des endémiques au niveau des étages supérieurs, en particulier de l'étage subalpin. Sur le plan phytosociologique, les résultats obtenus caractérisent le profil altitudinal des endémiques précédemment exposé (AESCHIMANN \& al., 2011b: 236-237), ou sur un total de 501 endémiques, 411 se rencontrent à l'étage subalpin (82\%), 318 au montagnard (63\%) et 295 à l'alpin (59\%). L'importance de l'étage montagnard est principalement liée aux endémiques des milieux rocheux de la classe des Asplenietea trichomanis, notamment ceux des sous-alliances endémiques de la bordure sud-orientale des Alpes (Physoplexido-Potentillenion) et de son extrémité sud-occidentale (Saxifragenion lingulatae), ainsi qu'aux endémiques des prés secs de la classe des FestucoBrometea. L'étage collinéen montre un profil d'endémiques comparable, mais où la prépondérance des Asplenietea trichomanis et des Festuco-Brometea est nettement plus marquée. A l'étage subalpin, les endémiques se concentrent dans les pelouses subalpines-alpines sur calcaire des Elyno-

Tableau 13. - Nombres de taxons endémiques (end.) et indigènes non endémiques (ind.) ayant potentiellement leur optimum sociologique dans une sélection de six classes (ou les syntaxons subordonnés), par étage de végétation ainsi que pour tous les étages confondus. En gras le plus grand nombre de chaque colonne, le maximum absolu dans l'un des étages et parmi les endémiques étant de plus encadré. Totaux des six classes, totaux généraux et pourcentages, ces derniers arrondis à l'unité.

\begin{tabular}{|c|c|c|c|c|c|c|c|c|c|c|c|c|c|}
\hline \multirow[t]{2}{*}{ № Synsystème } & \multirow[t]{2}{*}{ Classe } & \multicolumn{2}{|c|}{ Collinéen } & \multicolumn{2}{|c|}{ Montagnard } & \multicolumn{2}{|c|}{ Subalpin } & \multicolumn{2}{|c|}{ Alpin } & \multicolumn{2}{|c|}{ Nival } & \multicolumn{2}{|c|}{ C-M-S-A-N } \\
\hline & & End. & Ind. & End. & Ind. & End. & Ind. & End. & Ind. & End. & Ind. & End. & Ind. \\
\hline 3.2 & Asplenietea trichomanis & 56 & 103 & 86 & 107 & 92 & 100 & 51 & 67 & 11 & 11 & 113 & 141 \\
\hline 3.3 & Thlaspietea rotundifolii & 10 & 68 & 40 & 104 & 88 & 132 & 92 & 92 & 26 & 35 & 103 & 156 \\
\hline 10.2 & Elyno-Seslerietea variae & 16 & 44 & 48 & 137 & 93 & 172 & 70 & 158 & 2 & 11 & 93 & 173 \\
\hline 10.4 & Juncetea trifidi & 2 & 19 & 19 & 69 & 42 & 107 & 41 & 100 & 5 & 28 & 44 & 112 \\
\hline \multicolumn{2}{|c|}{ Totaux endémiques, indigènes } & 150 & 2871 & 318 & 2766 & 411 & 1937 & 295 & 803 & 46 & 106 & 501 & 3482 \\
\hline \multicolumn{2}{|c|}{$\%$ (6 classes sur total) } & 80 & 25 & 81 & 32 & 88 & 42 & 92 & 60 & 96 & 81 & 85 & 32 \\
\hline
\end{tabular}


Seslerietea variae, ainsi que dans les Asplenietea trichomanis (rochers) et dans les Thlaspietea rotundifolii (éboulis). Parmi les indigènes non endémiques, la classe des Festuco-Brometea demeure en revanche dominante au subalpin. A l'étage alpin, les endémiques des Thlaspietea rotundifolii (éboulis) et des Elyno-Seslerietea variae (pelouses calcaires) sont les plus nombreux; parmi les indigènes non endémiques, la classe des ElynoSeslerietea variae y devance nettement celle des Festuco-Brometea. A l'étage nival, la flore endémique est dominée par la classe des Thlaspietea rotundifolii (éboulis, 26 taxons sur 46, soit $56 \%$ ). De manière moins marquée, il en va de même au nival parmi les taxons indigènes non endémiques et l'on note de plus à cet étage que les taxons des pelouses sur silice des Juncetea trifidi sont 2,5 fois plus nombreux que ceux des pelouses sur calcaire des Elyno-Seslerietea variae, une situation inverse à celle observée à l'étage alpin, où les taxons des Elyno-Seslerietea variae sont 1,6 fois plus nombreux que ceux des Juncetea trifidi. Ces derniers résultats sont conformes avec l'augmentation précédemment démontrée (AESCHIMANN \& al., 2012b: 205) du pourcentage de taxons comptant pour les substrats siliceux, au passage de l'étage alpin à l'étage nival. Enfin, si la proportion de taxons indigènes non endémiques trouvant leur optimum sociologique dans les six classes sélectionnées augmente de $25 \%$ à $81 \%$ de l'étage collinéen à l'étage nival, la variation est bien moindre parmi les endémiques, passant en effet de $80 \%$ à $96 \%$ du collinéen au nival. Ces proportions confirment la tendance sténoèce des endémiques, mise en évidence à propos des milieux. La diminution en fonction de l'altitude de la diversité des optimums sociologiques des endémiques est très marquée dès l'étage subalpin, où les six classes du tableau 13 réunissent à elles seules près de neuf taxons sur dix.

\section{Conclusions}

\section{Milieux}

1. Parmi les 3482 taxons indigènes non endémiques recensés dans les Alpes, $70 \%$ sont présents dans les milieux herbacés et $57 \%$ dans les milieux nitrophiles. Les taux de présence sont inférieurs à $45 \%$ dans les cinq autres catégories principales de milieux et seulement $16 \%$ des indigènes sont exclusifs d'une des sept catégories principales. Les 501 taxons endémiques sont nettement plus sténoèces, puisque $81 \%$ d'entre eux se rencontrent dans les milieux rocheux ( $21 \%$ exclusifs) et $72 \%$ dans les milieux herbacés ( $5 \%$ exclusifs), alors que les taux de présence ne dépassent pas $25 \%$ dans les cinq autres catégories principales de milieux et que $27 \%$ des endémiques sont exclusifs d'une des sept catégories principales. La sténoécité des endémiques est confirmée par les proportions relevées dans les 35 catégories secondaires de milieux, car on en recense $63 \%$ sur les «rochers s.1.» ( $8 \%$ exclusifs), $59 \%$ dans les «éboulis s.1.» (3\% exclusifs) et $52 \%$ dans les «pelouses subalpines-alpines s.1.» (3\% exclusifs), alors que les proportions ne dépassent pas $25 \%$ dans les 32 autres milieux. L'amplitude écologique des endémiques est étroite, car $62 \%$ d'entre eux n'occupent qu'une à trois des catégories secondaires de milieux, contre $39 \%$ pour les indigènes non endémiques. Les 502 taxons xénophytes sont particulièrement nitrophiles, puisque $95 \%$ d'entre eux sont présents dans cette catégorie principale de milieux (souvent anthropiques) et que plus de $38 \%$ en sont même exclusifs. Cependant, avec 67 taxons comptant dans la catégorie secondaire des «rochers s.l.», soit 13\%, les xénophytes pourraient représenter une menace pour la flore endémique de ces milieux, surtout dans un contexte de réchauffement climatique.

2. Un gradient altitudinal est mis en évidence pour chaque catégorie principale de milieux. Ainsi, la proportion de taxons présents dans les milieux rocheux augmente très fortement de l'étage collinéen à l'étage nival, en passant de 39\% à 97\%. Au collinéen, c'est dans les milieux nitrophiles qu'on relève le pourcentage le plus élevé de taxons, soit $66 \%$. De l'étage montagnard à l'étage alpin, les milieux herbacés montrent les pourcentages de taxons les plus élevés, respectivement $68 \%, 75 \%$ et $82 \%$. Parmi les catégories secondaires, trois milieux rassemblent chacun plus de $50 \%$ des taxons à l'étage alpin et aucun autre ne se distingue avec plus de $25 \%$ : les «pelouses subalpines-alpines s.l.» rassemblent $68 \%$ des taxons, suivies des «éboulis s.1.» $(61 \%)$ et des «rochers s.l.» (54\%). A l'étage nival, trois milieux rassemblent chacun plus de $70 \%$ des taxons: les «éboulis s.1.» $(89 \%)$, les «rochers s.1.» (86\%) et les «pelouses subalpines-alpines s.1.» (72\%).

3. Le cortège floristique méditerranéen montre une proportion élevée de taxons dans les milieux nitrophiles, soit $78 \%$ (cultures, friches et milieux rudéraux). Le profil du cortège sud-européen-montagnard, dominant à l'étage alpin, est comparable à celui de cet étage. Dans le cortège arctico-alpin, les pourcentages élevés de taxons des milieux herbacés ( $81 \%$ ), rocheux $(65 \%)$ et marécageux (40\%) sont à l'image de la végétation du type toundra.

4. Parmi les douze familles réunissant chacune plus de 100 taxons dans les Alpes, les Cyperaceae montrent les proportions les plus élevées de taxons présents dans les milieux marécageux et aquatiques, respectivement $54 \%$ et $41 \%$. Les Fabaceae montrent le plus faible pourcentage dans les milieux marécageux, soit $4 \%$. La plus forte proportion de taxons présents dans les milieux rocheux est celle des Caryophyllaceae, soit $62 \%$, car cette famille compte $22 \%$ d'endémiques. Les Rosaceae montrent les pourcentages les plus élevés dans les milieux buissonneux et forestiers, respectivement $71 \%$ et $55 \%$. 
5. Parmi les quinze genres réunissant chacun au moins 40 taxons dans les Alpes, les Carex montrent les proportions les plus élevées de taxons présents dans les milieux marécageux et aquatiques, respectivement $55 \%$ et $30 \%$. Dans les milieux aquatiques, le genre Ranunculus égale ce dernier pourcentage. Les Saxifraga détiennent le plus haut pourcentage de taxons présents dans les milieux rocheux, soit $98 \%$, car ce genre compte $47 \%$ d'endémiques. Très sténoèces, les Saxifraga comptent moins de $50 \%$ de taxons présents dans chacune des six autres catégories principales de milieux.

6. Dans les milieux rocheux, ce sont les chaméphytes qui montrent la proportion la plus élevée de taxons, soit $81 \%$, ce qui confirme leur importance dans les habitats limitatifs. Pour les sept catégories principales de milieux, les profils des chaméphytes et des endémiques sont comparables. Les thérophytes dominent les milieux nitrophiles, avec $89 \%$ de taxons présents, surtout dans les cultures, les friches et les milieux rudéraux.

\section{Phytosociologie}

1. Quatre taxons endémiques sur cinq ont leur optimum sociologique au niveau de l'alliance ou de la sousalliance. Cela corrobore la sténoécité prononcée des endémiques, mise en évidence à propos des milieux. En revanche, un taxon indigène non endémique sur deux et seulement un xénophyte sur trois ont leur optimum à ces deux niveaux inférieurs.

2. Parmi les 3482 taxons indigènes non endémiques recensés dans les Alpes, près de $40 \%$ ont leur optimum sociologique dans les classes de végétation herbacée (appartenant aux formations 9, 10 et 11 du synsystème), ce qui confirme l'importance des milieux herbacés. Deux classes des mauvaises herbes des cultures et de végétation rudérale rassemblent $14 \%$ des taxons (les Stellarietea mediae et les Artemisietea vulgaris), en accord avec le second rang occupé par les milieux nitrophiles. La préférence des 501 taxons endémiques pour les milieux rocheux et d'éboulis se retrouve en phytosociologie, puisque trois classes rassemblent à elles seules 44\% des endémiques, les Asplenietea trichomanis (communautés végétales des fissures de rochers), les Thlaspietea rotundifolii (éboulis) et les KoelerioCorynephoretea (affleurements rocheux). Cependant, on note aussi la proportion très élevée d'endémiques ayant leur optimum dans les classes de végétation herbacée. Parmi les 502 taxons xénophytes, la forte tendance nitrophile est vérifiée, puisque deux classes rassemblent $45 \%$ des taxons, les Stellarietea mediae et les Artemisietea vulgaris.
3. Plus de la moitié des endémiques (51\%) ont leur optimum sociologique au niveau de neuf alliances seulement. La sténoécité est remarquable pour les milieux rocheux et les éboulis, puisque les premiers comprennent $20 \%$ des endémiques ayant leur optimum dans les deux alliances Potentillion caulescentis (calcaire) et Androsacion vandellii (silice), et les seconds $16 \%$ des endémiques dans les trois alliances Thlaspion rotundifolii (calcaire), Drabion hoppeanae (calcschistes) et Androsacion alpinae (silice). Au niveau des sous-alliances, les milieux rocheux regroupent encore $16 \%$ des endémiques. La présence de nombreux endémiques, dont certains étroitement sténoèces, dans quatre sous-alliances endémiques des Alpes justifie pleinement leur individualisation phytosociologique; ce sont le Physoplexido-Potentillenion, le Saxifragenion lingulatae, le Saxifragenion pedemontanae et le Caricenion austroalpinae.

4. Le patron cartographique du Physoplexido-Potentillenion, qui occupe les rochers calcaires, souvent dolomitiques, de la partie sud des Alpes orientales souligne à nouveau le «compartiment floristique» situé au sud de la faille périadriatique (ligne Adda - Drave), où dominent les roches sédimentaires, du lac de Côme à la Slovénie (AEschimAnN \& al., 2011a: 50; 2012b: 208). Trente-etun endémiques ont leur optimum sociologique dans le Physoplexido-Potentillenion, dont 13 sont étroitement sténoèces. Le patron du Caricenion austroalpinae (pelouses calcaires subalpines) est très comparable au précédent et 13 endémiques y trouvent leur optimum. A l'extrémité sud-occidentale des Alpes, le patron du Saxifragenion lingulatae, sous-alliance des rochers calcaires collinéenssubalpins, souligne le foyer d'endémisme des AlpesMaritimes et régions voisines (AESCHIMANN \& al., 2011a: 50). Vingt endémiques ont leur optimum dans le Saxifragenion lingulatae, dont huit sont étroitement sténoèces. Le patron du Saxifragenion pedemontanae (rochers siliceux subalpins-alpins) est comparable au précédent, mais son centre de gravité se situe sur le versant italien et six endémiques y trouvent leur optimum, dont un est étroitement sténoèce.

5. Par étage de végétation, la proportion de taxons la plus élevée atteinte par une classe est celle des Thlaspietea rotundifolii à l'étage nival, soit $40 \%$, ce qui corrobore le résultat maximal atteint par les «éboulis s.l.» au sein des 35 catégories secondaires de milieux, soit $89 \%$. La diversité des optimums sociologiques diminue nettement en fonction de l'altitude. Les tendances altitudinales des optimums sociologiques par classe traduisent une concentration des endémiques au niveau des étages supérieurs, en particulier de l'étage subalpin, un résultat conforme au profil altitudinal des endémiques précédemment exposé (AESCHIMANN \& al., 2011b: 236-237). 


\section{Remerciements}

Nous remercions le Fonds National Suisse de la recherche scientifique (FNS) de son soutien au projet «Contribution à l'étude synthétique de la diversité floristique des Alpes» ( ${ }^{\text {os }} 31-31244.91$ et 3100-031244), ainsi que toutes les personnes ayant collaboré au projet pour une Flore des Alpes. Nous remercions aussi Nicolas Wyler pour la production des cartes des Alpes par SIG, Yves Rasolofo pour ses conseils et expertises informatiques, ainsi que Nicolas Fumeaux pour la recherche de références bibliographiques.

\section{Références}

Aeschimann, D., K. Lauber, D. M. Moser \& J.-P. Theurillat (2004). Flora alpina. Haupt, Belin \& Zanichelli.

Aeschimann, D., N. Rasolofo \& J.-P. Theurillat (2011a). Analyse de la flore des Alpes. 1: historique et biodiversité. Candollea 66: 27-55.

Aeschimann, D., N. Rasolofo \& J.-P. Theurillat (2011b). Analyse de la flore des Alpes. 2: biodiversité et chorologie. Candollea 66: 225-253.

Aeschimann, D., N. Rasolofo \& J.-P. Theurillat (2012a). Analyse de la flore des Alpes. 3 : biologie et phénologie. Candollea 67 : 5-21.

Aeschimann, D., N. Rasolofo \& J.-P. Theurillat (2012b). Analyse de la flore des Alpes. 4: écologie. Candollea 67: 193-219.

BARBero, M. (1966). A propos de trois espèces rupicoles, endémiques des Alpes ligures. Bull. Soc. Bot. France 113: 330-341.

BARBero, M. (1967). L'endémisme dans les Alpes maritimes et ligures. Bull. Soc. Bot. France 114: 179-199.

Barni, E., G. Bacaro, S. Falzoi, F. Spanna \& C. Siniscalco (2012). Establishing climatic constraints shaping the distribution of alien plant species along the elevation gradient in the Alps. Pl. Ecol. 213: 757-767.

Becker, T., H. Dietz, R. Billeter, H. Buschmann \& P. J. Edwards (2005). Altitudinal distribution of alien plant species in the Swiss Alps. Perspect. Pl. Ecol. Evol. Syst. 7: 173-183.

Braun-Blanquet, J. (1948). La végétation alpine des Pyrénées orientales. Commun. Stat. Int. Géobot. Médit. Montpellier 98: 1-306.

Carraro, G., P. Gianoni, R. Mossi, F. Klötzli \& G.-R. Walther (2001). Observed changes in vegetation in relation to climate warming. In: Burga, C. A. \& A. Kratochwil (ed.), Biomonitoring: general and applied aspects on regional and global scales: 195205. Dordrecht, Kluwer.

CAsazza, C., G. BARberis \& L. Minuto (2005). Ecological characteristics and rarity of endemic plants of the Italian Maritime Alps. Biol. Conservation 123: 361-371.

DaINESE, M. \& L. BragazZA (2012). Plant traits across different habitats of the Italian Alps: a comparative analysis between native and alien species. Alpine Botany 122: 11-21.
DAnIN, A. (1999). Desert rocks as plant refugia in the Near East. Bot. Rev. 65: 93-170.

DAVIS, P. H. (1951). Cliff vegetation in the eastern Mediterranean. J. Ecol. 39: 63-93.

ELLENBERG, H. (1988). Vegetation ecology of Central Europe. Cambridge University Press.

Essl, F., M. Staudinger, O. Stöhr, L. Schratt-Ehrendorfer, W. RABITSCH \& H. NiKLFELD (2009). Distribution patterns, range size and niche breadth of Austrian endemic plants. Biol. Conservation 142: 2547-2558.

Feoli Chiapella, L. \& L. Poldini (1994). Prati e pascoli del Friuli (NE Italia) su substrati basici. Stud. Geobot. 13: 3-140.

Новонм, С. (2008). Ökologie und Verbreitung endemischer Gefässpflanzen in Europa. Tuexenia 28: 7-22.

Jeanmonod, D., A. Schlüssel \& J. Gamisans (2011a). Analyse de la flore Corse. Aspects biologiques. Candollea 66: 5-25.

JeAnmonod, D., A. Schlüssel \& J. Gamisans (2011b). Status and trends in the alien flora of Corsica. Bull. OEPP/EPPO Bull. 41 : 85-99.

Larson, D. W., U. Matthes \& P. E. Kelly (2000). Cliff ecology: pattern and process in cliff ecosystems. Cambridge University Press.

MARTINI, E. (1984). Lineamenti geobotanici delle Alpi Liguri e Maritime: endemismi e fitocenosi. Lav. Soc. Ital. Biogeogr. 9: 51-134.

MARTINI, E. (1997). Ricerche geobotaniche su Moehringia lebrunii Merxm. e Primula allionii Loisel. endemismi ristretti delle Alpi Maritime. In: Actes Coll. Ecol. Biogéogr. Alpines 1990. Rev. Valdôtaine Hist. Nat. 48, suppl.: 229-236.

Médail, F. \& R. Verlaque (1997). Ecological characteristics and rarity of endemic plants from southeast France and Corsica: implications for biodiversity conservation. Biol. Conservation 80: 269281.

Moradi, H., S. Fakheran, M. Peintinger, A. Bergamini, B. Schmid \& J. JoshI (2012). Profiteers of environmental change in the Swiss Alps: increase of thermophilous and generalist plants in wetland ecosystems within the last 10 years. Alpine Botany 122: 45-56.

MüLLER, T. (1997). Steinschutthalden als Wuchsorte von Reliktarten und als Ursprungsorte von Arten der Kulturlandschaft. Hoppea 58: 327-330.

Noble, V. \& K. Diadema (2011). La flore des Alpes-Maritimes et de la Principauté de Monaco. Originalité et diversité. Naturalia Publications, Turriers.

Ozenda, P. (1951). Eléments géographiques et endémisme dans les Alpes Maritimes et Ligures. Bull. Soc. Bot. France 97, sess. extr.: 141-156.

OZENDA, P. (1985). La végétation de la chaîne alpine dans l'espace montagnard européen. Masson.

PAWLOWSKI, B. (1970). Remarques sur l'endémisme dans la flore des Alpes et des Carpates. Vegetatio 21: 181-243.

QuÉZEL, P. (1951). Les groupements rupicoles calcicoles dans les Alpes-Maritimes. Leur signification biogéographique. Bull. Soc. Bot. France 97: 181-192. 
RABITSCH, W. \& F. EssL (ed.) (2009). Endemiten - Kostbarkeiten in Österreichs Pflanzen- und Tierwelt. Naturwissenschaftlicher Verein für Kärnten und Umweltbundesamt $\mathrm{GmbH}$.

RIouX, J. \& P. Quézel (1949). Contribution à l'étude des groupements rupicoles endémiques des Alpes-Maritimes. Vegetatio 2: 1-13.

Rusterholz, H.-P., D. WiRZ \& B. BAUR (2012). Garden waste deposits as a source for non-native plants in mixed deciduous forests. Appl. Veg. Sci. 15: 329-337.

Schroeter, C. (1926). Das Pflanzenleben der Alpen. Ed. 2. Raustein, Zürich.

Seipel, T., C. Kueffer, L. J. Rew, C. C. Daehler, A. Pauchard, B. J. Naylor, J. M. AleXander, P. J. Edwards, C. G. Parks, J. R. Arevalo, L. A. CaVieres, H. Dietz, G. Jakobs, K. McDougall, R. Otтo \& N. WALSh (2012). Processes at multiple scales affect richness and similarity of non-native plant species in mountains around the world. Global Ecol. Biogeogr. 21: 236-246.

Siniscalco, C., E. BARNi \& G. BACARo (2011). Non-native species distribution along the elevation gradient in the western Italian Alps. Pl. Biosyst. 145: 150-158.

SNOGERUP, S. (1971). Evolutionary and plant geographical aspects of chasmophytic communities. In: DAVIS, P. H., P. C. HARPER \& I. C. Hedge (ed.), Plant life of South-West Asia: 157-170. The Botanical Society of Edinburgh.

SteInGER, T., C. KöRNER \& B. SCHMID (1996). Long-term persistence in a changing climate: DNA analysis suggests very old ages of clones of alpine Carex curvula. Oecologia 105: 94-99.

SUTTER, R. (1962). Das Caricion austroalpinae. Ein neuer insubrischsüdalpiner Sesleria-Verband. Mitt. Ostalpin-Dinarischen Pflanzensoziol. Arbeitsgem. 2: 18-22.

SUTTER, R. (1969). Ein Beitrag zur Kenntnis der soziologischen Bindung süd-südostalpiner Reliktendemismen. Acta Bot. Croat. 28: 349-366.
TheuRILlat, J.-P., D. AEschimann, P. KÜPFER \& R. SPICHIGER (1995). The higher vegetation units of the Alps. Colloques Phytosociol. 23: $189-239$.

Theurillat, J.-P., F. Felber, P. Geissler, J.-M. Gobat, M. Fierz, A. Fischlin, P. KüPfer, A. Schlüssel, C. Velluti, G.-F. Zhao \& J. Williams (1998). Sensitivity of plant and soil ecosystems of the Alps to climate change. In: CEBon, P., U. Dahinden, H. C. DAVIES, D. M. IMBODEN \& C. C. Jäger (ed.), Views from the Alps. Regional perspectives on climate change: 225-308. MIT Press.

Theurillat, J.-P. \& A. GuISAN (2001). Potential impact of climate change on vegetation in the European Alps: a review. Climatic Change 50: 77-109; 53: 529-530.

TinNer, W. \& J.-P. TheURILLAT (2003). Uppermost limit, extent and fluctuations of the timberline ecotone in the Swiss Central Alps during the past 11,500 years. Arctic, Antarctic, Alp. Res. 35: 158169.

VIERHAPPER, F. (1924). Über endemische Alpenpflanzen. Alpenfreund: 147-148, 181-184.

VIERHAPPER, F. (1925). Über endemische Alpenpflanzen. Alpenfreund: 15-16, 47-48, 63-64, 79-80.

Walter, J., F. Essl, T. ENGLisch \& M. KieHN (2005). Aliens in Austria: habitat preferences and ecological effects. Neobiota 6: 13-25.

WALTHER, G.-R. (1999). Distribution and limits of evergreen broadleaved (laurophyllous) species in Switzerland. Bot. Helv. 109: 153-167.

WiersemA, G. (1989). Climate and vegetation characteristics of Ibex habitats in the European Alps. Mountain Res. Developm. 9: 119128. 